
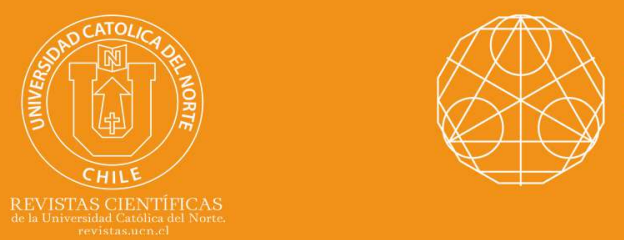

\title{
Strong convergence theorem for family of minimization and monotone inclusion problems in Hadamard spaces
}

Chinedu Izuchukwu' ${ }^{1}$ orcid.org/0000-0002-8262-8605

Godwin C. Ugwunnadi² ${ }^{2}$ orcid.org/0000-0002-2711-7888

Oluwatosin T. Mewomo ${ }^{3}$ [D orcid.org/0000-0003-0389-7469

University of KwaZulu-Natal, School of Mathematics Statistics and Computer Science, Durban, South Africa.

1曰izuchukwuc@ukzn.ac.za; ${ }^{\circledR}$ mewomoo@ukzn.ac.za

${ }^{2}$ University of Swaziland, Dept. of Mathematics, Kwaluseni, Swaziland.

ugwunnadi4u@yahoo.com

Received: May 2020 | Accepted: October 2020

\section{Abstract:}

In this paper, we introduce a modified Ishikawa-type proximal point algorithm for approximating a common solution of minimization problem, monotone inclusion problem and fixed point problem. We obtain a strong convergence of the proposed algorithm to a common solution of finite family of minimization problem, finite family of monotone inclusion problem and fixed point problem for asymptotically demicontractive mapping in Hadamard spaces. Numerical example is given to illustrate the applicability of our main result. Our results complement and extend some recent results in literature.

Keywords: CAT(0) spaces; Ishikawa-type proximal point algorithm; Monotone inclusion problem; Minimization problem; Fixed point problem; Asymptotically demicontractive mapping.

MSC (2020): 47H09, 47H10, 49J20, 49J40.

\section{Cite this article as (IEEE citation style):}

C. Izuchukwu, G. C. Ugwunnadi, and O. T. Mewomo, "Strong convergence theorem for family of minimization and monotone inclusion problems in Hadamard spaces", Proyecciones (Antofagasta, On line), vol. 40, no. 2, pp. 525-558, 2021, doi: 10.22199/issn.0717-6279-2021-02-0030

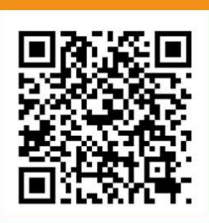

Article copyright: (c) 2021 Chinedu Izuchukwu, Godwin Chidi Ugwunnadi and Oluwatosin Temitope Mewomo. This is an open access article distributed under the terms of the Creative Commons License, which permits unrestricted use and distribution provided the original author and source are credited. 


\section{Introduction}

Let $(X, d)$ be a metric space. A geodesic path joining $x \in X$ to $y \in X$ is a mapping $c$ from a closed interval $[0, l] \subset \mathbf{R}$ to $X$ such that $c(0)=x, c(l)=y$ and $d\left(c(k), c\left(k^{\prime}\right)\right)=\left|k-k^{\prime}\right|$ for all $k, k^{\prime} \in[0, l]$. In this case, $c$ is called an isometry and $l=d(x, y)$. The image of $c$ is called a geodesic segment joining $x$ to $y$. When this image is unique, then it is denoted by $[x, y]$. The metric space $(X, d)$ is said to be a geodesic space if every two points of $X$ are joined by a geodesic and $(X, d)$ is said to be uniquely geodesic space if every two points of $X$ are joined by only one geodesic segment. A geodesic triangle $\Delta\left(x_{1}, x_{2}, x_{3}\right)$ in a geodesic space $(X, d)$ consist of three points $x_{1}, x_{2}, x_{3}$ in $X$ (known as the vertices of $\Delta$ ) and a geodesic segment between each pair of vertices (known as the edges of $\Delta$ ). A comparison triangle for the geodesic triangle $\Delta\left(x_{1}, x_{2}, x_{3}\right)$ in $(X, d)$ is a triangle $\bar{\Delta}\left(x_{1}, x_{2}, x_{3}\right):=\Delta\left(\bar{x}_{1}, \bar{x}_{2}, \bar{x}_{3}\right)$ in the Euclidean plane $\mathbf{R}^{2}$ such that $d\left(x_{i}, x_{j}\right)=d_{\mathbf{R}^{2}}\left(\bar{x}_{i}, \bar{x}_{j}\right)$ for $i, j \in\{1,2,3\}$. A metric space $(X, d)$ is called a $\operatorname{CAT}(0)$ space if it is geodesically connected and if every geodesic triangle in $X$ is at least as "thin" as its comparison triangle in the Euclidean plane $\mathbf{R}^{2}$. Furthermore, a geodesic space is a $\mathrm{CAT}(0)$ space if and only if it satisfies the following inequality, called the $(\mathrm{CN})$ inequality of Bruhat and Titis [14] (see [12]): If $x, y, z$ are points in $X$ and $y_{0}$ is the midpoint of the segment $[y, z]$, then

$$
d^{2}\left(x, y_{0}\right) \leq \frac{1}{2} d^{2}(x, y)+\frac{1}{2} d^{2}(x, z)-\frac{1}{4} d(y, z) .
$$

A complete $\operatorname{CAT}(0)$ space is called a Hadamard space. It is generally known that a $\operatorname{CAT}(0)$ space is a uniquely geodesic space. Examples of CAT(0) spaces includes: Euclidean spaces $\mathbf{R}^{n}$, Hilbert spaces, simply connected Riemannian manifolds of nonpositive sectional curvature, $\mathbf{R}$-trees, Hilbert ball [22], Hyperbolic spaces [51]. See [4, 12, 47] for more equivalent definitions and properties of $\operatorname{CAT}(0)$ spaces.

Let $(1-t) x \oplus t y$ denote the unique point $z$ in the geodesic segment joining $x$ to $y$ for each $x, y$ in a $\operatorname{CAT}(0)$ space such that $d(z, x)=t d(x, y)$ and $d(z, y)=(1-t) d(x, y)$, where $t \in[0,1]$. Let $[x, y]:=\{(1-t) x \oplus t y: t \in$ $[0,1]\}$, then a subset $C$ of $X$ is convex if $[x, y] \subseteq C$ for all $x, y \in C$. Let $X$ be a metric space and $C$ be a nonempty closed and convex subset of $X$. A point $x \in C$ is called a fixed point of a nonlinear mapping $T: C \rightarrow C$, if $T x=x$. The set of fixed points of $T$ is denoted by $\mathrm{F}(\mathrm{T})$. Many mathematical models of real-world problems arising from biology, economics, engineering, computer science, among others are usually expressed in the 
form of fixed point equation. Thus, the existence of solutions of these problems is equivalent to the existence of fixed points of some nonlinear mappings. The approximation of fixed points of nonlinear mappings is one of the most flourishing areas of research in nonlinear analysis and has continued to attract the interest of many researchers due to its extensive applications in diverse mathematical problems such as inverse problems, signal processing, image recovery, game theory, fuzzy theory, among others, see $[17,26,27,28,49,53,56,57,58]$ and the references therein. In metric spaces, fixed point theory begins with the Banach contraction mapping principle which guarantees the existence and uniqueness of fixed points of contraction mappings when the space is complete and the sequence of Pi- card iterates converges to the unique fixed point. Since then, the principle have been constantly improved and extended in many directions. In par- ticular, fixed point theorems in convex metric spaces have attracted the attention of many researchers (see, for example $[6,5$, $39,45])$.

Let $T: C \rightarrow C$ be a nonlinear mapping. $T$ is said to be

(i) L-Lipschitzian if there exists $L>0$ such that

$$
d(T x, T y) \leq L d(x, y) \forall x, y \in C,
$$

if $L \in[0,1)$, then $T$ is called contraction, while $T$ is called nonexpansive if $L=1$;

(ii) uniformly L-Lipschitzian if there exists $L>0$ such that

$$
d\left(T^{n} x, T^{n} y\right) \leq L d(x, y) \forall x, y \in C, n \geq 1
$$

(iii) asymptotically nonexpansive if there exists a sequence $\left\{k_{n}\right\}_{n=1}^{\infty} \subseteq$ $[1, \infty), \lim _{n \rightarrow \infty} k_{n}=1$ such that

$$
d\left(T^{n} x, T^{n} y\right) \leq k_{n} d(x, y) \forall n \geq 1 \text { and } x, y \in C ;
$$

(iv) asymptotically demicontractive if $F(T)=\emptyset$ and there exists a sequence $\left\{k_{n}\right\}_{n=1}^{\infty} \subseteq[1, \infty), \lim _{n \rightarrow \infty} k_{n}=1$ such that

$$
d^{2}\left(T^{n} x, y\right) \leq k_{n} d^{2}(x, y)+k d^{2}\left(x, T^{n} x\right)
$$

for some $k \in[0,1)$ and for all $n \geq 1, x \in C, y \in F(T)$; 
(v) $\left(\left\{\mu_{n}\right\},\left\{v_{n}\right\}, \phi\right)$-total asymptotically demicontractive if $F(T) \neq \emptyset$ and there exist a constant $k \in[0,1)$ and sequences $\left\{\mu_{n}\right\},\left\{v_{n}\right\} \subset[0, \infty)$ with $\mu_{n} \rightarrow 0, v_{n} \rightarrow 0$, and a strictly increasing continuous function $\phi:[0, \infty) \rightarrow[0, \infty)$ with $\phi(0)=0$ such that

$$
d^{2}\left(T^{n} x, y\right) \leq d^{2}(x, y)+\mu_{n} \phi(d(x, y))+k d^{2}\left(x, T^{n} x\right)+v_{n}
$$

for all $n \geq 1, x \in C, y \in F(T)$.

Let $\left\{x_{n}\right\}$ be a bounded sequence in $X$ and $r\left(.,\left\{x_{n}\right\}\right): X \rightarrow[0, \infty)$ be a continuous functional defined by $r\left(x,\left\{x_{n}\right\}\right)=n \rightarrow \infty \lim \sup d\left(x, x_{n}\right)$. The asymptotic radius of $\left\{x_{n}\right\}$ is given by $r\left(\left\{x_{n}\right\}\right):=\inf \left\{r\left(x,\left\{x_{n}\right\}\right): x \in X\right\}$ while the asymptotic center of $\left\{x_{n}\right\}$ is the set $A\left(\left\{x_{n}\right\}\right)=\{x \in X$ : $\left.r\left(x,\left\{x_{n}\right\}\right)=r\left(\left\{x_{n}\right\}\right)\right\}$. It is generally known that in a complete $\operatorname{CAT}(0)$ space, $A\left(\left\{x_{n}\right\}\right)$ consists of exactly one point. A sequence $\left\{x_{n}\right\}$ in $X$ is said to be $\Delta$-convergent to a point $x \in X$ if $A\left(\left\{x_{n_{k}}\right\}\right)=\{x\}$ for every subsequence $\left\{x_{n_{k}}\right\}$ of $\left\{x_{n}\right\}$. In this case, we write $\Delta-n \rightarrow \infty \lim x_{n}=x$ (see [21]). The concept of $\Delta$-convergence in metric spaces was first introduced and studied by Lim [42]. In 2008, Kirk and Panyanak [39] introduced and studied this concept in $\mathrm{CAT}(0)$ spaces and proved that it is very similar to the weak convergence in Banach space setting. Kirk [38] proved that every nonexpansive mapping defined on a nonempty closed convex and bounded subset of a $\operatorname{CAT}(0)$ space has a fixed point. In 2013, Kim [37] studied the Ishikawa-type iterative scheme for approximating fixed point of a completely continuous and uniformly L-Lipschitzian asymptotically demicontractive mapping in a complete $\mathrm{CAT}(0)$ space. Under some compactness condition, Kim [37] obtained a strong convergence result. Several other authors have also studied fixed point theory for nonlinear mappings in $\operatorname{CAT}(0)$ spaces (see, for example $[4,6,47]$.

Let $X$ be a $\operatorname{CAT}(0)$ space. A function $f: X \rightarrow(-\infty,+\infty]$ is called convex if

$$
f(\lambda x \oplus(1-\lambda) y) \leq \lambda f(x)+(1-\lambda) f(y) \forall x, y \in X, \lambda \in(0,1) .
$$

The function $f$ is called proper, if $D(f):=\{x \in X: f(x)<+\infty\} \neq \emptyset$, where $D(f)$ denotes the domain of $f$. A function $f: D(f) \rightarrow(-\infty, \infty]$ is said to be lower semi-continuous at a point $x \in D(f)$ if

$$
f(x) \leq \liminf _{n \rightarrow \infty} f\left(x_{n}\right),
$$

for each sequence $\left\{x_{n}\right\}$ which converges strongly to $x . f$ is said to be lower semicontinuous on $D(f)$ if it is lower semi-continuous at any point in $D(f)$. For any $\mu>0$, the resolvent of $f$ in $X$ is defined in [7] as 


$$
J_{\mu}^{f}(x)=\arg \min _{y \in X}\left[f(y)+\frac{1}{2 \mu} d^{2}(y, x)\right] .
$$

It was established in [32] that $J_{\mu}^{f}$ is well defined and that it is a non-expansive mapping for all $\mu>0$. For simplicity, we shall write $J_{\mu}$ for the resolvent of a proper convex and lower semi-continuous function $f$. The approximation of solutions of Minimization Problem (MP) has been of great interest in optimization theory, nonlinear analysis and geometry, see $[1,24]$. The MP is defined as follows: Find $x$ $\in X$ such that

$$
f(x)=\min _{y \in X} f(y) .
$$

We denote the solution set of problem (1.4) by $\operatorname{argmin}_{y \in X} f(y)$. In [7], it was shown that $F\left(J_{\mu}\right)$ coincides with $\operatorname{argmin}_{y \in X} f(y)$.

The Proximal Point Algorithm (PPA) is known to be one of the most effective and successful method for solving the MP (1.4). The PPA was introduced by Martinet [43] in 1970 and was further developed by Rockafellar [52] in Hilbert spaces as follows. Let $f$ be a proper convex and lower semi-continuous function defined on a Hilbert space $H$. The PPA is definedfor arbitrary $x_{1} \in H$ by

$$
x_{n+1}=\arg \min _{y \in H}\left(f(y)+\frac{1}{2 \mu_{n}}\left\|y-x_{n}\right\|^{2}\right),
$$

for each $n \geq 1$, where $\mu_{n}>0$ for all $n \geq 1$. It was proved in [52] that if $f$ has a minimizer in $H$ and $\sum_{n=1}^{\infty} \mu_{n}=\infty$, then the sequence $\left\{x_{n}\right\}$ converges weakly to a minimizer of $f$ (see also $[13,46]$ ). Güler [23] gave an example to show that the PPA does not necessarily converges strongly in general. To overcome this challenge, Kamimura and Takahashi [34] combined the PPA with the Halpern's algorithm, resulting to the following Halpern-type PPA, for which they obtained a strong convergence result for approximating solution of (1.4).

$$
\left\{\begin{array}{l}
u, x_{0} \in H, \\
x_{n+1}=\alpha_{n} u+\left(1-\alpha_{n}\right) J_{\mu_{n}} x_{n} .
\end{array}\right.
$$

The PPA was later introduced and studied in CAT(0) spaces by Bačák [9] for approximating a solution of MP in $\mathrm{CAT}(0)$ spaces. He proposed the following algorithm: For arbitrary $x_{1} \in X$, define the sequence $\left\{x_{n}\right\}$ by 


$$
x_{n+1}=\arg \min _{y \in X}\left(f(y)+\frac{1}{2 \mu_{n}} d^{2}\left(y, x_{n}\right)\right),
$$

for each $n \geq 1$, where $\mu_{n}>0$ for all $n \geq 1$. Under the conditions that $f$ has a minimizer in $X$ and $\sum_{n=1}^{\infty} \mu_{n}=\infty$, he proved that $\left\{x_{n}\right\} \Delta$-converges to a minimizer of $f$. In 2014, Bačák [8] studied a split version of the PPA for minimizing sum of convex functions in complete CAT(0) spaces. Very recently, Suparatulatorn et al. [54] propose the following Halpern-type PPA for approximating a common solution of a MP and fixed point problem fornonexpansive mapping in $\mathrm{CAT}(0)$ spaces:

$$
\left\{\begin{array}{l}
u, x_{1} \in X, \\
y_{n}=\arg \min _{y \in X}\left[f(y)+\frac{1}{2 \mu_{n}} d^{2}\left(y, x_{n}\right)\right], \\
x_{n+1}=\alpha_{n} u \oplus\left(1-\alpha_{n}\right) T y_{n} n \geq 1,
\end{array}\right.
$$

where $\left\{\alpha_{n}\right\} \subset(0,1)$ and $\mu_{n} \geq \mu>0$. They obtained a strong convergenceresult under some mild conditions. For more results on PPA for MP, see[7, 10, 24, 59] and the references therein.

In 2008, Breg and Nikolaev [11] introduced the concept of quailinearization mapping in CAT(0) spaces. They denoted a pair $(a, b) \in X \times X$ by $\overrightarrow{a b}$ which they called a vector and defined a mapping $\langle.,\rangle:.(X \times X) \times(X \times X) \rightarrow \mathbf{R}$ by

$$
\langle\overrightarrow{a b}, \overrightarrow{c d}\rangle={ }_{2}^{\mathrm{t}}\left(d^{2}(a, d)+d^{2}(b, c)-d^{2}(a, c)-d^{2}(b, d)\right),(a, b, c, d \in X)
$$

called the quasilinearization mapping. It is easy to verify that $\langle\overrightarrow{a b}, \overrightarrow{a b}\rangle=d^{2}(a, b),\langle\overrightarrow{b a}, \overrightarrow{c d}\rangle=-\langle\overrightarrow{a b}, \overrightarrow{c d}\rangle,\langle\overrightarrow{a b}, \overrightarrow{c d}\rangle=\langle\overrightarrow{a e}, \overrightarrow{c d}\rangle+\langle\overrightarrow{e b}, \overrightarrow{c d}\rangle$ and $\langle\overrightarrow{a b}, \overrightarrow{c d}\rangle=\langle\overrightarrow{c d}, \overrightarrow{a b}\rangle$ for all $a, b, c, d, e \in X$. It has been established that a geodesically connected metric space is a CAT(0) space if and only if it satisfies the Cauchy-Schwartz inequality (see [11]). Recall that the space $X$ is said to satisfy the Cauchy-Swartz inequality if $\langle\overrightarrow{a b}, \overrightarrow{c d}\rangle \leq d(a, b) d(c, d) \forall a, b, c, d \in$ $X$.

In 2010, Ahmadi Kakavandi and Amini [3] introduced the concept of dual space in a complete $\operatorname{CAT}(0)$ space $X$ by first introducing the concept of pseudometric space which they defined as the space $\mathbf{R} \times X \times X$ together with a pseudometric $D$ defined by

$$
D((t, a, b),(s, c, d))=L(\Theta(t, a, b)-\Theta(s, c, d)), \quad(t, s \in \mathbf{R}, a, b, c, d \in X),
$$


where $\Theta: \mathbf{R} \times X \times X \rightarrow C(X, \mathbf{R})$ is defined as $\Theta(t, a, b)(x)=t\langle\overrightarrow{a b}, \overrightarrow{a x}\rangle,(t \in$ $\mathbf{R}, a, b, x \in X)$ and $L(\varphi)=\sup \left\{\frac{\varphi(x)-\varphi(y)}{d(x, y)}: x, y \in X, x=y\right\}$ for any function $\varphi: X \rightarrow \mathbf{R}$. Furthermore, $D$ defines an equivalence relation on $\mathbf{R} \times X \times X$, where the equivalence class of $(t, a, b)$ is

$[t \overrightarrow{a b}]:=\{s \overrightarrow{c d}: D((t, a, b),(s, c, d))=0\}$. Then, $X^{*}=\{[t \overrightarrow{a b}]:(t, a, b) \in$ $\mathbf{R} \times X \times X\}$ is a metric space with $D$ as the metric. The pair $\left(X^{*}, D\right)$ is called the dual space of $(X, d)$. The dual space $X^{*}$ acts on $X \times X$ by $\left\langle x^{*}, \overrightarrow{x y}\right\rangle=t\langle\overrightarrow{a b}, \overrightarrow{x y}\rangle, \quad x^{*}=[t \overrightarrow{a b}] \in X^{*}, x, y \in X$ (see [36]). It was shown in [3] that the dual of a closed and convex subset of a Hilbert space $H$ with nonempty interior is $H$ and $t(b-a) \equiv[t \overrightarrow{a b}]$ for all $t \in \mathbf{R}, a, b \in H$.

Let $X$ be a complete CAT(0) space and $X^{*}$ be its dual space. A multivalued operator $A: X \rightarrow 2^{X^{*}}$ with domain $\mathbf{D}(A):=\{x \in X: A x=\emptyset\}$ is monotone if and only if for all $x, y \in \mathbf{D}(A), x^{*} \in A x, y^{*} \in A y$, we have

$$
\left\langle x^{*}-y^{*}, \overrightarrow{y x}\right\rangle \geq 0 \quad(\text { see }[36]) .
$$

The resolvent of the operator $A$ of order $\lambda>0$ is the multivalued mapping $J_{\lambda}^{A}: X \rightarrow 2^{X}$ defined in [36] as

$$
J_{\lambda}^{A}(x):=\left\{z \in X \mid\left[\frac{1}{\lambda} \overrightarrow{z x}\right] \in A z\right\} .
$$

The operator $A$ satisfies the range condition if for every $\lambda>0, \mathbf{D}\left(J_{\lambda}^{A}\right)=X$ (see [36]). For simplicity, we shall write $J_{\lambda}$ for the resolvent of a monotone operator $A$.

Monotone operator theory is an area of research in nonlinear and convex analysis that has continued to attract the interest of many researchers due to the role it plays in diverse mathematical problems such as optimization problems, equilibrium problems, variational inequality problems, saddle point problems, among others (see $[2,29,30,31,47,55]$ and the ref-erences therein). Many of these problems can be modelled as a Monotone Inclusion Problem (MIP) which is considered the most important problems in monotone operator theory (see $[36,50]$ and the references therein). Let $A: X \rightarrow 2^{X^{*}}$ be a monotone operator, the MIP is

to find

$x \in \mathbf{D}(A)$ such that $0 \in A x$.

The solution set of problem (1.9) is denoted by $A^{-1}(0)$, which is known to be closed and convex (see [50], Remark 3.1). 
The PPA is one of the most popular method for finding solution of (1.9). In 1976, Rockafellar [52] studied the following PPA for approximating asolution of MIP (1.9) in Hilbert space $H$, for $x_{0} \in H$

$$
x_{n-1}-x_{n} \in \lambda_{n} A\left(x_{n}\right),
$$

where $\left\{\lambda_{n}\right)$ is a sequence of positive real numbers. Rockafellar [52] proved that the sequence $\left\{x_{n}\right\}$ generated by Algorithm (1.10) is weakly convergentto a solution of (1.9), provided $\lambda_{n} \geq \lambda>0$ for each $n \geq 1$. Since then, numerous authors have developed and studied different methods for findingsolutions of (1.9) in Hilbert spaces and Banach spaces (see $[15,34,35,48]$ and the references therein). Recently, researchers are beginning to study MIP in CAT(0) spaces. In 2016, Khatibzadeh and Ranjbar [36] introducedand studied the following PPA in CAT(0) spaces for approximating a solu-tion of MIP, for which they obtained both strong and $\Delta$-convergence result,using the algorithm

$$
\left\{\begin{array}{l}
x_{0} \in X, \\
x_{n}=J_{\lambda_{n}} x_{n-1},
\end{array}\right.
$$

where $\left\{\lambda_{n}\right\}$ is a sequence of positive real numbers such that $\sum_{n=1}^{\infty} \lambda_{n}=$ $\infty$. Very recently, Ranjbar and Khatibzadeh [50] proposed the following Mann-type and Halpern-type PPA in complete CAT(0) spaces for finding a solution of MIP:

$$
\left\{\begin{array}{l}
x_{0} \in X \\
x_{n+1}=\alpha_{n} x_{n} \oplus\left(1-\alpha_{n}\right) J_{\lambda_{n}} x_{n}
\end{array}\right.
$$

and

$$
\left\{\begin{array}{l}
u, x_{0} \in X, \\
x_{n+1}=\alpha_{n} u \oplus\left(1-\alpha_{n}\right) J_{\lambda_{n}} x_{n},
\end{array}\right.
$$

where $\left\{\lambda_{n}\right\} \subset(0, \infty)$ and $\left\{\alpha_{n}\right\} \subset[0,1]$. They obtained a $\Delta$-convergence result using the Mann-type PPA while they obtained a strong convergence result using the Halpern-type PPA.

In this paper, we propose the Ishikawa-type PPA for approximating a common solution of finite family of MP, finite family of MIP and fixed point problem for asymptotically demicontractive mapping in Hadamard spaces. Our results extend and complement the results of Kim [37], Bačák [9], Suparatulatorn et al. [54], Khatibzadeh and Ranjbar [36] and Ranjbar and Khatibzadeh [50]. 


\section{Preliminary}

We state some known and useful results which will be needed in the proof of our main results.

Let $X$ be a $\operatorname{CAT}(0)$ space. Then, for all $x, y, z \in X$ and $t, s \in[0,1]$, the following hold:

(i) $d(t x \oplus(1-t) y, z) \leq t d(x, z)+(1-t) d(y, z)$, (see [47, 59]),

(ii) $d^{2}(t x \oplus(1-t) y, z) \leq t d^{2}(x, z)+(1-t) d^{2}(y, z)-t(1-t) d^{2}(x, y)$, (see $[47,59])$

(iii) $d(t x \oplus(1-t) y, s x \oplus(1-s) y) \leq|t-s| d(x, y)$, (see [16]),

(iv) $d(t x \oplus(1-t) y, t x \oplus(1-t) z) \leq(1-t) d(y, z)$, (see [38]).

[19] Let $X$ be a $\operatorname{CAT}(0)$ space and $a, b, c \in X$. Then for each $t \in[0,1]$, $d^{2}(t a \oplus(1-t) b, c) \leq t^{2} d^{2}(a, c)+(1-t)^{2} d^{2}(b, c)+2 t(1-t)\langle\overrightarrow{a c}, \overrightarrow{b c}\rangle$.

$[59,41]$ Every bounded sequence in a complete $\mathrm{CAT}(0)$ space has a $\Delta$-convergence subsequence.

[33] Let $X$ be a complete CAT(0) space, $\left\{x_{n}\right\}$ be a sequence in $X$ and $x \in X$. Then $\left\{x_{n}\right\} \Delta$-converges to $x$ if and only if $n \rightarrow \infty \lim \sup \left\langle\overrightarrow{x_{n}} \vec{x}, \overrightarrow{y x}\right\rangle \leq$ $0 \forall y \in X$.

[20] Let $X$ be a complete $\mathrm{CAT}(0)$ space and $T: X \rightarrow X$ be a nonexpansive mapping, then the conditions that $\left\{x_{n}\right\} \Delta$-converges to $x$ and $\left\{d\left(x_{n}, T x_{n}\right)\right\}$ converges strongly to 0 , implies that $x=T x$.

[25] Let $C$ be a closed and convex subset of a complete CAT(0) space $X$ and $T: C \rightarrow C$ be a uniformly $L$-Lipschitzian and $\left(\{\mu\},\left\{v_{n}\right\}, \phi\right)$-total asymptotically demicontractive mapping with $F(T)=\emptyset$. If $\left\{x_{n}\right\}$ is a bounded sequence in $C$ such that $\Delta-n \rightarrow \infty \lim x_{n}=p$ and $n \rightarrow \infty \lim d\left(x_{n}, T x_{n}\right)=$ 0 . Then $T p=p$.

Remark 2.1. If $\phi(\lambda)=\lambda^{2}$ and $v_{n}=0$ for each $n \geq 1$, then in Lemma 2.6, $T$ is an asymptotically demicontractive mapping.

[62]. Let $\left\{a_{n}\right\}$ be a sequence of non-negative real numbers satisfying

$$
a_{n+1} \leq\left(1-\alpha_{n}\right) a_{n}+\alpha_{n} \delta_{n}+\gamma_{n}, \quad n \geq 0,
$$


where $\left\{\alpha_{n}\right\},\left\{\delta_{n}\right\}$ and $\left\{\gamma_{n}\right\}$ satisfy the following conditions:

(i) $\left\{\alpha_{n}\right\} \subset[0,1], \Sigma_{n=0}^{\infty} \alpha_{n}=\infty$,

(ii) $\lim \sup _{n \rightarrow \infty} \delta_{n} \leq 0$,

(iii) $\gamma_{n} \geq 0(n \geq 0), \Sigma_{n=0}^{\infty} \gamma_{n}<\infty$.

Then $\lim _{n \rightarrow \infty} a_{n}=0$.

[44]. Let $\left\{a_{n}\right\}$ be a sequence of real numbers such that there exists a subsequence $\left\{n_{j}\right\}$ of $\{n\}$ with $a_{n_{j}}<a_{n_{j}+1} \forall j \in \mathbf{N}$. Then there exists a nondecreasing sequence $\left\{m_{k}\right\} \subset \mathbf{N}$ such that $m_{k} \rightarrow \infty$ and the following properties are satisfied by all (sufficiently large) numbers $k \in \mathbf{N}$ :

$$
a_{m_{k}} \leq a_{m_{k}+1} \text { and } a_{k} \leq a_{m_{k}+1} .
$$

In fact, $m_{k}=\max \left\{i \leq k: a_{i}<a_{i+1}\right\}$.

[40] Let $(X, d)$ be a complete $\mathrm{CAT}(0)$ space and $f: X \rightarrow(-\infty, \infty]$ be a proper convex and lower semi-continuous function. Then, for all $x, y \in X$ and $\mu>0$, we have

(2.1) $\frac{1}{2 \mu} d^{2}\left(J_{\mu} x, y\right)-\frac{1}{2 \mu} d^{2}(x, y)+\frac{1}{2 \mu} d^{2}\left(x, J_{\mu} x\right)+f\left(J_{\mu} x\right) \leq f(y)$.

Definition 2.2. Let $X$ be a complete $C A T(0)$ space and $C$ be a nonempty closed and convex subset of $X$. A mapping $T: C \rightarrow C$ is said to be a firmly nonexpansive mapping (see [36]) if

$$
d^{2}(T x, T y) \leq\langle\overrightarrow{T x T y}, \overrightarrow{x y}\rangle \forall x, y \in C .
$$

From Cauchy-Schwartz inequality, it is clear that the class of nonexpasive mappings is more general than the class of firmly nonexpansive mappings (see [36]).

[36] Let $X$ be a $\operatorname{CAT}(0)$ space and $J_{\lambda}$ be the resolvent of a multivalued operator $A: X \rightarrow 2^{X^{*}}$ of order $\lambda$. Then, we have the following;

(i) for any $\lambda>0, \quad F\left(J_{\lambda}\right)=A^{-1}(0)$,

(ii) if $A$ is monotone, then $J_{\lambda}$ is a single-valued and a nonexpansive mapping. 


\section{Main Results}

We make the following remark which is a consequence of Theorem 2.12.

Remark 3.1. If $X$ is a $C A T(0)$ space and $J_{\lambda}$ is the resolvent of a monotone operator $A: X \rightarrow 2^{X^{*}}$ of order $\lambda>0$, then

$$
d^{2}\left(u, J_{\lambda} x\right)+d^{2}\left(J_{\lambda} x, x\right) \leq d^{2}(u, x),
$$

for all $u \in A^{-1}(0)$ and $x \in \mathbf{D}\left(J_{\lambda}\right)$.

Proof. Indeed for any $u \in A^{-1}(0), x \in \mathbf{D}\left(J_{\lambda}\right)$ and $\lambda>0$, we obtain from Theorem 2.12 and Definition 2.11 that

$$
\begin{aligned}
d^{2}\left(J_{\lambda} x, u\right) & \leq\left\langle\overrightarrow{J_{\lambda} x u}, \overrightarrow{x u}\right\rangle \\
& =\frac{1}{2}\left(d^{2}\left(J_{\lambda} x, u\right)+d^{2}(u, x)-d^{2}\left(J_{\lambda} x, x\right)\right),
\end{aligned}
$$

which implies

$$
d^{2}\left(u, J_{\lambda} x\right)+d^{2}\left(J_{\lambda} x, x\right) \leq d^{2}(u, x) .
$$

Let $X$ be a Hadamard space and $X^{*}$ be its dual space. For each $i=$ $1,2, \ldots N$, let $A_{i}: X \rightarrow 2^{X^{*}}$ be multivalued monotone mappings that satisfy the range condition and let $J_{\lambda}^{i}$ be the resolvent of $A_{i}$ for any $\lambda>0$. For each $j=1,2, \ldots, m$, let $f_{j}: X \rightarrow(-\infty, \infty]$ be proper convex and lower semi continuous functions and $J_{\mu}^{j}$ be the resolvent of $f_{j}$ for any $\mu>0$. Then,

$$
F\left(\Phi_{\lambda}^{N} \circ \Psi_{\mu}^{m}\right)=\left(\cap_{i=1}^{N} A_{i}^{-1}(0)\right) \cap\left(\cap_{j=1}^{m} \arg \min _{y \in X} f_{j}(y)\right),
$$

where $\Phi_{\lambda}^{N}=J_{\lambda}^{N} \circ J_{\lambda}^{N-1} \circ \ldots \circ J_{\lambda}^{2} \circ J_{\lambda}^{1}, \quad \Phi_{\lambda}^{0}=I$ and $\Psi_{\mu}^{m}=J_{\mu}^{N} \circ J_{\mu}^{m-1} \circ \ldots \circ$ $J_{\mu}^{2} \circ J_{\mu}^{1}, \quad \Phi_{\mu}^{0}=I$.

Proof. Observe that $\left(\cap_{i=1}^{N} A_{i}^{-1}(0)\right) \cap\left(\cap_{j=1}^{m} \arg \min _{y \in X} f_{j}(y)\right)=F\left(J_{\lambda}^{N}\right) \cap$ $F\left(J_{\lambda}^{N-1}\right) \cap \ldots \cap F\left(J_{\lambda}^{2}\right) \cap F\left(J_{\lambda}^{1}\right) \cap F\left(J_{\mu}^{m}\right) \cap F\left(J_{\mu}^{m-1}\right) \cap \ldots \cap F\left(J_{\mu}^{2}\right) \cap F\left(J_{\mu}^{1}\right)$. Clearly, $\left(\cap_{i=1}^{N} A_{i}^{-1}(0)\right) \cap\left(\cap_{j=1}^{m} \arg \min _{y \in X} f_{j}(y)\right) \subseteq F\left(\Phi_{\lambda}^{N} \circ \Psi_{\mu}^{m}\right)$.

We now show that $F\left(\Phi_{\lambda}^{N} \circ \Psi_{\mu}^{m}\right) \subseteq\left(\cap_{i=1}^{N} A_{i}^{-1}(0)\right) \cap\left(\cap_{j=1}^{m} \arg \min _{y \in X} f_{j}(y)\right)$. 
Let $x \in F\left(\Phi_{\lambda}^{N} \circ \Psi_{\mu}^{m}\right)$ and $y \in\left(\left(\cap_{i=1}^{N} A_{i}^{-1}(0)\right) \cap\left(\cap_{j=1}^{m} \arg \min _{y \in X} f_{j}(y)\right)\right)$.

Then,

$$
\begin{aligned}
d^{2}(x, y) & =d^{2}\left(\Phi_{\lambda}^{N} \Psi_{\mu}^{m} x, \Phi_{\lambda}^{N} \Psi_{\mu}^{m} y\right) \\
& \leq d^{2}\left(\Phi_{\lambda}^{N-1} \Psi_{\mu}^{m} x, \Phi_{\lambda}^{N-1} \Psi_{\mu}^{m} y\right) \\
& =d^{2}\left(\Phi_{\lambda}^{N-1} \Psi_{\mu}^{m} x, y\right) .
\end{aligned}
$$

From Remark 3.1, we obtain that

$$
\begin{aligned}
d^{2}\left(J_{\lambda}^{N}\left(\Phi_{\lambda}^{N-1} \Psi_{\mu}^{m} x\right), \Phi_{\lambda}^{N-1} \Psi_{\mu}^{m} x\right) & \leq d^{2}\left(\Phi_{\lambda}^{N-1} \Psi_{\mu}^{m} x, y\right)-d^{2}\left(J_{\lambda}^{N}\left(\Phi_{\lambda}^{N-1} \Psi_{\mu}^{m} x\right), y\right) \\
& \vdots \\
& \leq d^{2}(x, y)-d^{2}\left(\Phi_{\lambda}^{N} \Psi_{\mu}^{m} x, y\right) \\
& =d^{2}(x, y)-d^{2}(x, y),
\end{aligned}
$$

which implies

$$
\Phi_{\lambda}^{N} \Psi_{\mu}^{m} x=\Phi_{\lambda}^{N-1} \Psi_{\mu}^{m} x
$$

From Remark 3.1 and (3.1), we obtain

$$
\begin{aligned}
d^{2}\left(J_{\lambda}^{N-1}\left(\Phi_{\lambda}^{N-2} \Psi_{\mu}^{m} x\right), \Phi_{\lambda}^{N-2} \Psi_{\mu}^{m} x\right) & \leq d^{2}\left(\Phi_{\lambda}^{N-2} \Psi_{\mu}^{m} x, y\right)-d^{2}\left(J_{\lambda}^{N-1}\left(\Phi_{\lambda}^{N-2} \Psi_{\mu}^{m} x\right), y\right) \\
& \vdots \\
& \leq d^{2}(x, y)-d^{2}\left(\Phi_{\lambda}^{N-1} \Psi_{\mu}^{m} x, y\right) \\
& \leq d^{2}\left(\Phi_{\lambda}^{N-1} \Psi_{\mu}^{m} x, y\right)-d^{2}\left(\Phi_{\lambda}^{N-1} \Psi_{\mu}^{m} x, y\right),
\end{aligned}
$$

which implies

$$
\Phi_{\lambda}^{N-1} \Psi_{\mu}^{m} x=\Phi_{\lambda}^{N-2} \Psi_{\mu}^{m} x
$$

where the last inequality follows from (3.1).

Also, from Remark 3.1 and (3.1), we have 


$$
\begin{aligned}
d^{2}\left(\Phi_{\lambda}^{N-2} \Psi_{\mu}^{m} x, \Phi_{\lambda}^{N-3} \Psi_{\mu}^{m} x\right) & \leq d^{2}\left(\Phi_{\lambda}^{N-3} \Psi_{\mu}^{m} x, y\right)-d^{2}\left(\Phi_{\lambda}^{N-2} \Psi_{\mu}^{m} x, y\right) \\
& \vdots \\
& \leq d^{2}(x, y)-d^{2}\left(\Phi^{N-2} \Psi_{\mu}^{m} x, y\right) \\
& \leq d^{2}\left(\Phi_{\lambda}^{N-1} \Psi_{\mu}^{m} x, y\right)-d^{2}\left(\Phi_{\lambda}^{N-1} \Psi_{\mu}^{m} x, y\right),
\end{aligned}
$$

which implies

$$
\Phi_{\lambda}^{N-2} \Psi_{\mu}^{m} x=\Phi_{\lambda}^{N-3} \Psi_{\mu}^{m} x .
$$

Continuing in this manner, we obtain that

$$
\Phi_{\lambda}^{N-3} \Psi_{\mu}^{m} x=\Phi_{\lambda}^{N-4} \Psi_{\mu}^{m} x=\ldots=\Phi_{\lambda}^{2} \Psi_{\mu}^{m} x=\Phi_{\lambda}^{1} \Psi_{\mu}^{m} x=\Phi_{\lambda}^{0} \Psi_{\mu}^{m} x=\Psi_{\mu}^{m} x
$$

Furthermore, we obtain from Lemma 2.10 and (3.1) that

$\frac{1}{2 \mu} d^{2}\left(\Psi_{\mu}^{m} x, y\right)-\frac{1}{2 \mu} d^{2}\left(\Psi_{\mu}^{m-1} x, y\right)+\frac{1}{2 \mu} d^{2}\left(\Psi_{\mu}^{m-1} x, \Psi_{\mu}^{m} x\right)+f\left(\Psi_{\mu}^{m} x\right) \leq f(y)$.

Since $f(y) \leq f\left(\Psi_{\mu}^{m} x\right)$, we have that

$$
\begin{aligned}
d^{2}\left(\Psi_{\mu}^{m} x, \Psi_{\mu}^{m-1} x\right) & \leq d^{2}\left(\Psi_{\mu}^{m-1} x, y\right)-d^{2}\left(\Psi_{\mu}^{m} x, y\right) \\
& \vdots \\
& \leq d^{2}(x, y)-d^{2}\left(\Phi_{\lambda}^{N-1} \Psi_{\mu}^{m} x, y\right) \\
& \leq d^{2}\left(\Phi_{\lambda}^{N-1} \Psi_{\mu}^{m} x, y\right)-d^{2}\left(\Phi_{\lambda}^{N-1} \Psi_{\mu}^{m} x, y\right),
\end{aligned}
$$

which implies

$$
\Psi_{\mu}^{m} x=\Psi_{\mu}^{m-1} x .
$$

Following similar argument as above, we obtain that 
(3.7) $\Psi_{\mu}^{m-1} x=\Psi_{\mu}^{m-2} x=\Psi_{\mu}^{m-3} x=\ldots=\Psi_{\mu}^{2} x=\Psi_{\mu}^{1} x=\Psi_{\mu}^{0} x=x$.

Hence, from (3.2), (3.3), (3.4), (3.5), (3.6) and (3.7), we have

$$
\begin{array}{r}
\Phi_{\lambda}^{N} \Psi_{\mu}^{m} x=\Phi_{\lambda}^{N-1} \Psi_{\mu}^{m} x=\ldots=\Phi_{\lambda}^{1} \Psi_{\mu}^{m} x=\Psi_{\mu}^{m} x=\Psi_{\mu}^{m-1} x \\
=\Psi_{\mu}^{m-2} x=\ldots=\Psi_{\mu}^{2} x=\Psi_{\mu}^{1} x=x .
\end{array}
$$

From (3.8), we obtain

$$
x=J_{\mu}^{1} x .
$$

From (3.8) and (3.9), we obtain

$$
x=\Psi_{\mu}^{2} x=J_{\mu}^{2}\left(J_{\mu}^{1} x\right)=J_{\mu}^{2} x .
$$

Continuing in this manner, we obtain

$$
x=J_{\mu}^{1} x=J_{\mu}^{2} x=\ldots=J_{\mu}^{m-1} x=J_{\mu}^{m} x=J_{\lambda}^{1} x=\ldots=J_{\lambda}^{N-1} x=J_{\lambda}^{N} x .
$$

Thus, it follows from (3.11) that

$$
F\left(\Phi_{\lambda}^{N} \circ \Psi_{\mu}^{m}\right) \subseteq\left(\cap_{i=1}^{N} A_{i}^{-1}(0)\right) \cap\left(\cap_{j=1}^{m} \arg \min _{y \in X} f_{j}(y)\right),
$$

which completes the proof.

Let $X$ be a Hadamard space and $X^{*}$ be its dual space. Let $A_{i}: X \rightarrow$ $2^{X^{*}}, i=1,2, \ldots N$ be multivalued monotone mappings that satisfy the range condition and $f_{j}: X \rightarrow(-\infty, \infty], j=1,2, \ldots, m$ be proper convex and lower semi continuous functions. Let $T: X \rightarrow X$ be a uniformly $L$-Lipschitzian and asymptotically demicontractive mapping with constant $k \in(0,1)$ and sequence $\left\{k_{n}\right\}_{n=1}^{\infty} \subseteq[1, \infty)$ such that $\sum_{n=1}^{\infty}\left(k_{n}^{2}-1\right)<\infty$. 
Suppose that $\Gamma:=F(T) \cap\left(\cap_{i=1}^{N} A_{i}^{-1}(0)\right) \cap\left(\cap_{j=1}^{m} \arg \min _{y \in X} f_{j}(y)\right) \neq \emptyset$. Let $u, x_{1} \in X$ be arbitrary and the sequence $\left\{x_{n}\right\}$ be generated by

$$
\left\{\begin{array}{l}
v_{n}=\left(1-t_{n}\right) x_{n} \oplus t_{n} u \\
u_{n}=\Phi_{\lambda}^{N} \circ \Psi_{\mu}^{m}\left(v_{n}\right), \\
y_{n}=\left(1-\beta_{n}\right) u_{n} \oplus \beta_{n} T^{n} u_{n}, \\
x_{n+1}=\left(1-\alpha_{n}\right) u_{n} \oplus \alpha_{n} T^{n} y_{n}, n \geq 1,
\end{array}\right.
$$

where $\Phi_{\lambda}^{N}=J_{\lambda}^{N} \circ J_{\lambda}^{N-1} \circ \ldots \circ J_{\lambda}^{2} \circ J_{\lambda}^{1}, \Phi_{\lambda}^{0}=I, \Psi_{\mu}^{m}=J_{\mu}^{N} \circ J_{\mu}^{m-1} \circ \ldots \circ$ $J_{\mu}^{2} \circ J_{\mu}^{1}, \Phi_{\mu}^{0}=I, \lambda, \mu \in(0, \infty)$ and $\left\{t_{n}\right\},\left\{\beta_{n}\right\},\left\{\alpha_{n}\right\}$ are sequences in $(0,1)$ satisfying the following conditions:

$\mathrm{C} 1: n \rightarrow \infty \lim t_{n}=0$,

C2: $\sum_{n=1}^{\infty} t_{n}=\infty$,

C3: $0<\epsilon \leq \alpha_{n} \leq k \beta_{n}<\beta_{n} \leq b<\frac{2}{\left(\frac{k+\delta}{k}\right)+\sqrt{\left(\frac{k+\delta}{k}\right)^{2}+4 L^{2}}}$, where $\delta:=$ $n \geq 1 \sup k_{n}$,

$\mathrm{C} 4: n \rightarrow \infty \lim \frac{k_{n}^{2}-1}{t_{n}}=0$.

Then $\left\{x_{n}\right\}$ converges strongly to an element of $\Gamma$.

Proof. We first observe that since $T$ is uniformly $L$-Lipschitzian, we obtain from (3.12) that

$$
\begin{aligned}
d\left(T^{n} u_{n}, T^{n} y_{n}\right) & \leq L d\left(u_{n}, y_{n}\right) \\
& =L d\left(u_{n},\left(1-\beta_{n}\right) u_{n} \oplus \beta_{n} T^{n} u_{n}\right) \\
& \leq L \beta_{n} d\left(u_{n}, T^{n} u_{n}\right) .
\end{aligned}
$$

Let $p \in \Gamma$, then from (3.12) and (3.13), we have

$$
\begin{aligned}
d^{2}\left(T^{n} y_{n}, p\right) \leq & k_{n} d^{2}\left(y_{n}, p\right)+k d^{2}\left(y_{n}, T^{n} y_{n}\right) \\
= & k_{n} d^{2}\left(\left(1-\beta_{n}\right) u_{n} \oplus \beta_{n} T^{n} u_{n}, p\right)+k d^{2}\left(y_{n}, T^{n} y_{n}\right) \\
\leq & k_{n}\left(1-\beta_{n}\right) d^{2}\left(u_{n}, p\right)+k_{n} \beta_{n} d^{2}\left(T^{n} u_{n}, p\right)-k_{n} \beta_{n}\left(1-\beta_{n}\right) d^{2}\left(u_{n}, T^{n} u_{n}\right) \\
& +k d^{2}\left(\left(1-\beta_{n}\right) u_{n} \oplus \beta_{n} T^{n} u_{n}, T^{n} y_{n}\right) \\
\leq & k_{n}\left(1-\beta_{n}\right) d^{2}\left(u_{n}, p\right)+k_{n} \beta_{n} d^{2}\left(T^{n} u_{n}, p\right)-k_{n} \beta_{n}\left(1-\beta_{n}\right) d^{2}\left(u_{n}, T^{n} u_{n}\right)
\end{aligned}
$$




$$
\begin{aligned}
& +k\left(1-\beta_{n}\right) d^{2}\left(u_{n}, T^{n} y_{n}\right)+k \beta_{n} d^{2}\left(T^{n} u_{n}, T^{n} y_{n}\right)-k \beta_{n}\left(1-\beta_{n}\right) d^{2}\left(T^{n} u_{n}, u_{n}\right) \\
\leq & k_{n}\left(1-\beta_{n}\right) d^{2}\left(u_{n}, p\right)+k_{n} \beta_{n} d^{2}\left(T^{n} u_{n}, p\right)-k_{n} \beta_{n}\left(1-\beta_{n}\right) d^{2}\left(u_{n}, T^{n} u_{n}\right) \\
& +k\left(1-\beta_{n}\right) d^{2}\left(u_{n}, T^{n} y_{n}\right)+k L^{2} \beta_{n}^{3} d^{2}\left(u_{n}, T^{n} u_{n}\right)-k \beta_{n}\left(1-\beta_{n}\right) d^{2}\left(T^{n} u_{n}, u_{n}\right) \\
\leq & k_{n}\left(1-\beta_{n}\right) d^{2}\left(u_{n}, p\right)+k_{n} \beta_{n}\left[k_{n} d^{2}\left(u_{n}, p\right)+k d^{2}\left(u_{n}, T^{n} u_{n}\right)\right]-k_{n} \beta_{n}\left(1-\beta_{n}\right) d^{2}\left(u_{n}, T^{n}\right. \\
& +k\left(1-\beta_{n}\right) d^{2}\left(u_{n}, T^{n} y_{n}\right)+k L^{2} \beta_{n}^{3} d^{2}\left(u_{n}, T^{n} u_{n}\right)-k \beta_{n}\left(1-\beta_{n}\right) d^{2}\left(T^{n} u_{n}, u_{n}\right) \\
= & \left(k_{n}-k_{n} \beta_{n}+k_{n}^{2} \beta_{n}\right) d^{2}\left(u_{n}, p\right)-\beta_{n}\left[\left(1-\beta_{n}\right)\left(k_{n}+k\right)-k L^{2} \beta_{n}^{2}-k k_{n}\right] d^{2}\left(u_{n}, T^{n} u_{n}\right) \\
& +k\left(1-\beta_{n}\right) d^{2}\left(u_{n}, T^{n} y_{n}\right) \\
\leq & {\left[k_{n}^{2}\left(1-\beta_{n}\right)+k_{n}^{2} \beta_{n}\right] d^{2}\left(u_{n}, p\right)+k\left(1-\beta_{n}\right) d^{2}\left(u_{n}, T^{n} y_{n}\right) } \\
& -\beta_{n}\left[\left(1-\beta_{n}\right)\left(k_{n}+k\right)-k L^{2} \beta_{n}^{2}-k k_{n}\right] d^{2}\left(u_{n}, T^{n} u_{n}\right) \\
= & k_{n}^{2} d^{2}\left(u_{n}, p\right)+k\left(\mathbf{3 . 1 4} \beta_{n}\right) d^{2}\left(u_{n}, T^{n} y_{n}\right)-\beta_{n}\left[\left(1-\beta_{n}\right)\left(k_{n}+k\right)-k L^{2} \beta_{n}^{2}-k k_{n}\right] d^{2}\left(u_{n},\right.
\end{aligned}
$$

Also, from (3.12), (3.14) and condition C3, we obtain

$$
\begin{aligned}
d^{2}\left(x_{n+1}, p\right) \leq & \left(1-\alpha_{n}\right) d^{2}\left(u_{n}, p\right)+\alpha_{n} d^{2}\left(T^{n} y_{n}, p\right)-\alpha_{n}\left(1-\alpha_{n}\right) d^{2}\left(u_{n}, T^{n} y_{n}\right) \\
\leq & \left(1-\alpha_{n}\right) d^{2}\left(u_{n}, p\right)+\alpha_{n} k_{n}^{2} d^{2}\left(u_{n}, p\right)+k \alpha_{n}\left(1-\beta_{n}\right) d^{2}\left(u_{n}, T^{n} y_{n}\right) \\
& -\alpha_{n} \beta_{n}\left[\left(1-\beta_{n}\right)\left(k_{n}+k\right)-k L^{2} \beta_{n}^{2}-k k_{n}\right] d^{2}\left(u_{n}, T^{n} u_{n}\right)-\alpha_{n}\left(1-\alpha_{n}\right) d^{2}\left(u_{n}, T^{n} y_{n}\right) \\
\leq & k_{n}^{2} d^{2}\left(u_{n}, p\right)-\alpha_{n}\left[(1-k)+\left(k \beta_{n}-\alpha_{n}\right)\right] d^{2}\left(u_{n}, T^{n} y_{n}\right) \\
& -\alpha_{n} \beta_{n}\left[\left(1-\beta_{n}\right)\left(k_{n}+k\right)-k L^{2} \beta_{n}^{2}-k k_{n}\right] d^{2}\left(u_{n}, T^{n} u_{n}\right) \\
\leq & k_{n}^{2} d^{2}\left(u_{(23} \cdot \text {. p5 } 5\right) \alpha_{n} \beta_{n}\left[\left(1-\beta_{n}\right)\left(k_{n}+k\right)-k L^{2} \beta_{n}^{2}-k k_{n}\right] d^{2}\left(u_{n}, T^{n} u_{n}\right) \\
\leq & {\left[1+\left(k_{n}^{3} \cdot 161\right)\right] d^{2}\left(u_{n}, p\right) } \\
= & {\left[1+\left(k_{n}^{2}-1\right)\right] d^{2}\left(\Phi_{\lambda}^{N} \Psi_{\mu}^{m} v_{n}, p\right) } \\
\leq & {\left[1+\left(k_{n}^{2}-1\right)\right] d^{2}\left(\Phi_{\lambda}^{N-1} \Psi_{\mu}^{m} v_{n}, p\right) } \\
\vdots & \\
\leq & {\left[1+\left(k_{n}^{2}-1\right)\right] d^{2}\left(\Psi_{\mu}^{m} v_{n}, p\right) } \\
\leq & {\left[1+\left(k_{n}^{2}-1\right)\right] d^{2}\left(\Psi_{\mu}^{m-1} v_{n}, p\right) } \\
\vdots & \\
\leq & {\left[1+\left(k_{n}^{2}-1\right)\right] d^{2}\left(v_{n}, p\right) } \\
\leq & {\left[1+\left(k_{n}^{2}-1\right)\right]\left[\left(1-t_{n}\right) d^{2}\left(x_{n}, p\right)+t_{n} d^{2}(u, p)\right] }
\end{aligned}
$$




$$
\begin{aligned}
& \leq\left[1+\left(k_{n}^{2}-1\right)\right] \max \left\{d^{2}\left(x_{n}, p\right), d^{2}(u, p)\right\} \\
& \vdots \\
& \leq \prod_{r=1}^{n}\left[1+\left(k_{r}^{2}-1\right)\right] \max \left\{d^{2}\left(x_{1}, p\right), d^{2}(u, p)\right\} .
\end{aligned}
$$

Since $\sum_{n=1}^{\infty}\left(k_{n}^{2}-1\right)<\infty$, then we have that $\left\{x_{n}\right\}$ is bounded. Consequently, $\left\{u_{n}\right\},\left\{v_{n}\right\}$ are all bounded.

From (3.12) and condition C1, we have that

$$
\lim _{n \rightarrow \infty} d\left(v_{n}, x_{n}\right) \leq \lim _{n \rightarrow \infty} t_{n} d\left(u, x_{n}\right)=0 .
$$

We now divide our proof into two cases.

Case 1: Suppose that $\left\{d^{2}\left(x_{n}, p\right)\right\}$ is monotonically non-increasing, then $n \rightarrow \infty \lim \left\{d^{2}\left(x_{n}, p\right)\right\}$ exists. Consequently,

$$
\lim _{n \rightarrow \infty}\left[d^{2}\left(x_{n+1}, p\right)-d^{2}\left(x_{n}, p\right)\right]=0 .
$$

From Remark 3.1 and (3.15), we obtain

$$
\begin{aligned}
d^{2}\left(u_{n}, \Phi_{\lambda}^{N-1} \Psi^{m} v_{n}\right) & =d^{2}\left(J_{\lambda}^{N}\left(\Phi_{\lambda}^{N-1} \Psi^{m}\right) v_{n}, \Phi_{\lambda}^{N-1} \Psi^{m} v_{n}\right) \\
& \leq d^{2}\left(\Phi_{\lambda}^{N-1} \Psi_{\mu}^{m} v_{n}, p\right)-d^{2}\left(J_{\lambda}^{N}\left(\Phi_{\lambda}^{N-1} \Psi^{m}\right) v_{n}, p\right) \\
& \vdots \\
& \leq d^{2}\left(v_{n}, p\right)-\frac{1}{k_{n}^{2}} d^{2}\left(x_{n+1}, p\right) \\
& \leq d^{2}\left(v_{n}, x_{n}\right)+d^{2}\left(x_{n}, p\right)+2 d\left(v_{n}, x_{n}\right) d\left(x_{n}, p\right)-\frac{1}{k_{n}^{2}} d^{2}\left(x_{n+1}, p\right) \\
(3.19) & =d^{2}\left(v_{n}, x_{n}\right)+2 d\left(v_{n}, x_{n}\right) d\left(x_{n}, p\right)+\left[d^{2}\left(x_{n}, p\right)-\frac{1}{k_{n}^{2}} d^{2}\left(x_{n+1}, p\right)\right] .
\end{aligned}
$$

Since $n \rightarrow \infty \lim k_{n}=1$ and $n \rightarrow \infty \lim d\left(x_{n}, p\right)$ exists, we have from (3.17) and (3.19) that

$$
\lim _{n \rightarrow \infty} d^{2}\left(u_{n}, \Phi_{\lambda}^{N-1} \Psi^{m} v_{n}\right)=0 .
$$

Again, from Lemma 2.1 and Remark 3.1, we obtain 


$$
\begin{aligned}
d^{2}\left(\Phi_{\lambda}^{N-1} \Psi_{\mu}^{m} v_{n}, \Phi_{\lambda}^{N-2} \Psi_{\mu}^{m} v_{n}\right) \leq & d^{2}\left(\Phi_{\lambda}^{N-2} \Psi_{\mu}^{m} v_{n}, p\right)-d^{2}\left(\Phi_{\lambda}^{N-1} \Psi^{m} v_{n}, p\right) \\
\vdots & \\
\leq & d^{2}\left(v_{n}, p\right)-d^{2}\left(u_{n}, p\right) \\
\leq & d^{2}\left(v_{n}, p\right)-\frac{1}{k_{n}^{2}} d^{2}\left(x_{n+1}, p\right) \\
\leq & d^{2}\left(v_{n}, x_{n}\right)+2 d\left(v_{n}, x_{n}\right) d\left(x_{n}, p\right) \\
& +\left[d^{2}\left(x_{n}, p\right)-\frac{1}{k_{n}^{2}} d^{2}\left(x_{n+1}, p\right)\right] \rightarrow 0, \text { as } n \rightarrow \infty .
\end{aligned}
$$

Continuing in the same manner, we have that

$$
\underset{n \rightarrow \infty}{\left(\lim 22 d^{2}\right.}\left(\Phi_{\lambda}^{N-2} \Psi_{\mu}^{m} v_{n}, \Phi_{\lambda}^{N-3} \Psi_{\mu}^{m} v_{n}\right)=\ldots=\lim _{n \rightarrow \infty} d^{2}\left(\Phi_{\lambda}^{1} \Psi_{\mu}^{m} v_{n}, \Psi_{\mu}^{m} v_{n}\right)=0
$$

Also, from Lemma 2.10, we have

$$
\frac{1}{2 \mu} d^{2}\left(\Psi_{\mu}^{m} v_{n}, p\right)-\frac{1}{2 \mu} d^{2}\left(\Psi_{\mu}^{m-1} v_{n}, p\right)+\frac{1}{2 \mu} d^{2}\left(\Psi_{\mu}^{m-1} v_{n}, \Psi_{\mu}^{m} p\right)+f\left(\Psi_{\mu}^{m} v_{n}\right) \leq f(p) .
$$

Since $f(p) \leq f\left(\Psi_{\mu}^{m} v_{n}\right)$, we have that

$$
\begin{aligned}
d^{2}\left(\Psi_{\mu}^{m} v_{n}, \Psi_{\mu}^{m-1} v_{n}\right) \leq & d^{2}\left(\Psi_{\mu}^{m-1} v_{n}, p\right)-d^{2}\left(\Psi_{\mu}^{m} v_{n}, p\right) \\
\vdots & \\
\leq & d^{2}\left(v_{n}, p\right)-d^{2}\left(u_{n}, p\right) \\
\leq & d^{2}\left(v_{n}, p\right)-\frac{1}{k_{n}^{2}} d^{2}\left(x_{n+1}, p\right) \\
\leq & d^{2}\left(v_{n}, x_{n}\right)+2 d\left(v_{n}, x_{n}\right) d\left(x_{n}, p\right) \\
& +\left[d^{2}\left(x_{n}, p\right)-\frac{1}{k_{n}^{2}} d^{2}\left(x_{n+1}, p\right)\right] \rightarrow 0, \text { as } n \rightarrow \infty
\end{aligned}
$$

Using similar argument as above, we can show that

$$
\left.\lim _{n \rightarrow \infty} d^{2}\left(\Psi_{\mu}^{m\left(3.24_{4}\right)}\right), \Psi_{\mu}^{m-2} v_{n}\right)=\ldots=\lim _{n \rightarrow \infty} d^{2}\left(\Psi_{\mu}^{2} v_{n}, \Psi_{\mu}^{1} v_{n}\right)=\lim _{n \rightarrow \infty} d^{2}\left(\Psi_{\mu}^{1} v_{n}, v_{n}\right)=0 .
$$

Thus, 


$$
\begin{aligned}
d\left(u_{n}, v_{n}\right) \leq & d\left(u_{n}, \Phi_{\lambda}^{N-1} \Psi_{\mu}^{m} v_{n}\right)+d\left(\Phi_{\lambda}^{N-1} \Psi_{\mu}^{m} v_{n}, \Phi_{\lambda}^{N-2} \Psi_{\mu}^{m} v_{n}\right) \\
& +\ldots+d\left(\Phi_{\lambda}^{1} \Psi_{\mu}^{m} v_{n}, \Psi_{\mu}^{m} v_{n}\right)+d\left(\Psi_{\mu}^{m} v_{n}, \Psi_{\mu}^{m-1} v_{n}\right) \\
& +d\left(\Psi_{\mu}^{m-1} v_{n}, \Psi_{\mu}^{m-2} v_{n}\right)+\ldots+d\left(\Psi_{\mu}^{1} v_{n}, v_{n}\right),
\end{aligned}
$$

which implies from (3.20), (3.21), (3.22), (3.23) and (3.24), that

$$
\lim _{n \rightarrow \infty} d\left(u_{n}, v_{n}\right)=\lim _{n \rightarrow \infty} d\left(\Phi_{\lambda}^{N} \Psi_{\mu}^{m} v_{n}, v_{n}\right)=0 .
$$

From (3.17) and (3.25), we obtain

$$
\lim _{n \rightarrow \infty} d\left(u_{n}, x_{n}\right)=0 .
$$

Since $\left\{d^{2}\left(x_{n}, p\right)\right\}$ and $\left\{k_{n}\right\}$ are bounded, then there exists $M>0$ such that

$$
M:=n \geq 1 \sup \left\{d^{2}\left(x_{n}, p\right), k_{n}^{2}\right\} .
$$

Thus, from (3.15), we obtain

$$
\begin{aligned}
& d^{2}\left(x_{n+1}, p\right)-d^{2}\left(x_{n}, p\right) \\
\leq & k_{n}^{2} d^{2}\left(u_{n}, p\right)-d^{2}\left(x_{n}, p\right) \\
& -\alpha_{n} \beta_{n}\left[\left(1-\beta_{n}\right)\left(k_{n}+k\right)-k L^{2} \beta_{n}^{2}-k k_{n}\right] d^{2}\left(u_{n}, T^{n} u_{n}\right) \\
\leq & k_{n}^{2} d^{2}\left(u_{n}, x_{n}\right)+k_{n}^{2} d^{2}\left(x_{n}, p\right)+2 k_{n}^{2} d\left(u_{n}, x_{n}\right) d\left(x_{n}, p\right)-d^{2}\left(x_{n}, p\right) \\
& -\alpha_{n} \beta_{n}\left[\left(1-\beta_{n}\right)\left(k_{n}+k\right)-k L^{2} \beta_{n}^{2}-k k_{n}\right] d^{2}\left(u_{n}, T^{n} u_{n}\right) \\
\leq & M d^{2}\left(u_{n}, x_{n}\right)+2 M^{2} d\left(u_{n}, x_{n}\right)+\left(k_{n}^{2}-1\right) M \\
& -\alpha_{n} \beta_{n}\left[\left(1-\beta_{n}\right)\left(k_{n}+k\right)-k L^{2} \beta_{n}^{2}-k k_{n}\right] d^{2}\left(u_{n}, T^{n} u_{n}\right) .
\end{aligned}
$$

Since $\sum_{n=1}^{\infty}\left(k_{n}^{2}-1\right)<\infty$, then $n \rightarrow \infty \lim \left(k_{n}^{2}-1\right)=0$. Thus, letting

$$
\delta_{n}=\alpha_{n} \beta_{n}\left[\left(1-\beta_{n}\right)\left(k_{n}+k\right)-k L^{2} \beta_{n}^{2}-k k_{n}\right],
$$

we obtain from (3.27) and (3.26) that

$$
\begin{aligned}
\delta_{n} d^{2}\left(u_{n}, T^{n} u_{n}\right) \leq & M d^{2}\left(u_{n}, x_{n}\right)+2 M^{2} d\left(u_{n}, x_{n}\right)+M\left(k_{n}^{2}-1\right) \\
& +d^{2}\left(x_{n}, p\right)-d^{2}\left(x_{n+1}, p\right) \rightarrow 0, \text { as } n \rightarrow \infty .
\end{aligned}
$$


From condition C3, we obtain that $2 k-b(k+\delta)>k b \sqrt{\left(\frac{k+\delta}{k}\right)^{2}+4 L^{2}}$. This implies that

$$
[2 k-b(k+\delta)]^{2}>4 L^{2} k^{2} b^{2}+b^{2}(k+\delta)^{2},
$$

which further implies that

$$
k-b k-b \delta-k L^{2} b^{2}>0 .
$$

Thus,

$$
\begin{aligned}
\delta_{n} & =\alpha_{n} \beta_{n}\left[\left(k_{n}+k\right)-k k_{n}-\beta_{n}\left(k+k_{n}\right)-k L^{2} \beta_{n}^{2}\right] \\
& >\epsilon^{2}\left[\left(k_{n}+k\right)-k_{n}-\beta_{n}\left(k+k_{n}\right)-k L^{2} \beta_{n}^{2}\right] \\
& >\epsilon^{2}\left[k-b k-b k_{n}-k L^{2} b^{2}\right] \\
& >\epsilon^{2}\left[k-b k-b \delta-k L^{2} b^{2}\right]>0 .
\end{aligned}
$$

Hence, from (3.28), we obtain

$$
\lim _{n \rightarrow \infty} d\left(u_{n}, T^{n} u_{n}\right)=0 .
$$

From (3.12) and condition C3, we obtain

$$
\lim _{n \rightarrow \infty} d\left(y_{n}, u_{n}\right) \leq \lim _{n \rightarrow \infty} \beta_{n} d\left(T^{n} u_{n}, u_{n}\right)=0 .
$$

Since $T$ is uniformly $L$-Lipschitzian, we obtain from (3.29) and (3.30) that

$$
\begin{aligned}
d\left(x_{n+1}, y_{n}\right) \leq & \left(1-\alpha_{n}\right) d\left(u_{n}, y_{n}\right)+\alpha_{n} d\left(T^{n} y_{n}, y_{n}\right) \\
\leq & \left(1-\alpha_{n}\right) d\left(u_{n}, y_{n}\right)+\alpha_{n} d\left(T^{n} y_{n}, T^{n} u_{n}\right) \\
& +\alpha_{n}\left[d\left(T^{n} u_{n}, u_{n}\right)+d\left(y_{n}, u_{n}\right)\right] \\
\leq & \left(1-\alpha_{n}\right) d\left(u_{n}, y_{n}\right)+\alpha_{n} L d\left(y_{n}, u_{n}\right) \\
& +\alpha_{n}\left[d\left(T^{n} u_{n}, u_{n}\right)+d\left(y_{n}, u_{n}\right)\right] \rightarrow 0, \text { as } n \rightarrow \infty .
\end{aligned}
$$

From (3.26), (3.30) and (3.31), we have 


$$
\lim _{n \rightarrow \infty} d\left(x_{n+1}, x_{n}\right)=0 .
$$

Again, since $T$ is uniformly $L$-Lipschitzian, we obtain

$$
\begin{aligned}
d\left(u_{n}, T u_{n}\right) \leq & d\left(u_{n}, T^{n} u_{n}\right)+d\left(T^{n} u_{n}, T u_{n}\right) \\
\leq & d\left(u_{n}, T^{n} u_{n}\right)+L d\left(T^{n-1} u_{n}, u_{n}\right) \\
\leq & d\left(u_{n}, T^{n} u_{n}\right)+L d\left(T^{n-1} u_{n}, T^{n-1} u_{n-1}\right) \\
& +L d\left(T^{n-1} u_{n-1}, u_{n-1}\right)+L d\left(u_{n-1}, u_{n}\right) \\
\leq & d\left(u_{n}, T^{n} u_{n}\right)+L^{2} d\left(u_{n}, u_{n-1}\right) \\
& +L d\left(T^{n-1} u_{n-1}, u_{n-1}\right)+L d\left(u_{n-1}, u_{n}\right) \\
\leq & d\left(u_{n}, T^{n} u_{n}\right)+L d\left(T^{n-1} u_{n-1}, u_{n-1}\right) \\
& +\left(L^{2}+L\right)\left[d\left(u_{n}, x_{n}\right)+d\left(x_{n}, x_{n-1}\right)+d\left(x_{n-1}, u_{n-1}\right)\right],
\end{aligned}
$$

which implies from (3.26), (3.29) and (3.32) that

$$
\lim _{n \rightarrow \infty} d\left(u_{n}, T u_{n}\right)=0 .
$$

Since $\left\{x_{n}\right\}$ is bounded and $X$ is a Hadamard space, then from Lemma 2.3, there exists a subsequence $\left\{x_{n_{k}}\right\}$ of $\left\{x_{n}\right\}$ such that $\Delta-k \rightarrow \infty \lim x_{n_{k}}=z$. It follows from (3.26) that there exists a subsequence $\left\{u_{n_{k}}\right\}$ of $\left\{u_{n}\right\}$ such that $\Delta-k \rightarrow \infty \lim u_{n_{k}}=z$. Thus, from (3.33) and Lemma 2.6, we obtain that $z \in F(T)$. Also, since $\Phi_{\lambda}^{N} \circ \Psi_{\mu}^{m}$ is the composition of nonexpansive mappings, it implies that $\Phi_{\lambda}^{N} \circ \Psi_{\mu}^{m}$ is nonexpansive. Thus, it follows from (3.25) and Lemma 2.5 that $z \in F\left(\Phi_{\lambda}^{N} \circ \Psi_{\mu}^{m}\right)$. Hence, by Lemma 3.2, we obtain that $z \in\left(\cap_{i=1}^{N} A_{i}^{-1}(0)\right) \cap\left(\cap_{j=1}^{m} \arg \min _{y \in X} f_{j}(y)\right)$. Therefore, $z \in \Gamma$. Furthermore, for arbitrary $u \in X$, we have from Lemma 2.4 that

$$
\limsup _{n \rightarrow \infty}\left\langle\overrightarrow{u z}, \overrightarrow{x_{n} \vec{z}}\right\rangle \leq 0,
$$

which implies from condition $\mathrm{C} 1$ that

$$
n \rightarrow \infty \lim \sup \left(t_{n} d^{2}(u, z)+2\left(1-t_{n}\right)\left\langle\overrightarrow{u z}, \overrightarrow{x_{n} z}\right\rangle\right) \leq 0 .
$$


Next, we show that $\left\{x_{n}\right\}$ converges strongly to $z$. Since $\left\{u_{n}\right\}$ is bounded, there exists $M^{\prime}>0$ such that $d^{2}\left(u_{n}, z\right) \leq M^{\prime} \forall n \geq 1$. Thus, from (3.16) and Lemma 2.2, we obtain

$$
\begin{aligned}
d^{2}\left(x_{n+1}, z\right) & \leq d^{2}\left(u_{n}, z\right)+\left(k_{n}^{2}-1\right) d^{2}\left(u_{n}, z\right) \\
& \leq d^{2}\left(u_{n}, z\right)+\left(k_{n}^{2}-1\right) M^{\prime} \\
& \leq d^{2}\left(\Phi_{\lambda}^{N-1} \Psi_{\mu}^{m} v_{n}, z\right)+\left(k_{n}^{2}-1\right) M^{\prime} \\
& \vdots \\
& \leq d^{2}\left(\Psi_{\mu}^{m} v_{n}, z\right)+\left(k_{n}^{2}-1\right) M^{\prime} \\
& \vdots \\
& \leq d^{2}\left(v_{n}, z\right)+\left(k_{n}^{2}-1\right) M^{\prime} \\
& \leq\left(1-t_{n}\right)^{2} d^{2}\left(x_{n}, z\right)+t_{n}^{2} d^{2}(u, z)+2 t_{n}\left(1-t_{n}\right)\left\langle\overrightarrow{u z}, \overrightarrow{x_{n} z}\right\rangle+\left(k_{n}^{2}-1\right) M^{\prime} \\
& \leq\left(1-t_{n}\right) d^{2}\left(x_{n}, z\right)+t_{n}\left(t_{n} d^{2}(u, z)+2\left(1-t_{n}\right)\left\langle\overrightarrow{u z}, \overrightarrow{x_{n} z}\right\rangle\right)+\left(k_{n}^{2}-1\right) M^{\prime} .
\end{aligned}
$$

Since $\sum_{n=1}^{\infty}\left(k_{n}^{2}-1\right) M^{\prime}<\infty$, it then follows from (3.35), (3.36) and Lemma 2.8 that $\left\{x_{n}\right\}$ converges strongly to $z$.

Case 2: Suppose that $\left\{d^{2}\left(x_{n}, p\right)\right\}$ is monotonically non-decreasing. Then, there exists a subsequence $\left\{d^{2}\left(x_{n_{i}}, p\right)\right\}$ of $\left\{d^{2}\left(x_{n}, p\right)\right\}$ such that $d^{2}\left(x_{n_{i}}, p\right)<$ $d^{2}\left(x_{n_{i}+1}, p\right)$ for all $i \in \mathbf{N}$. Thus, by Lemma 2.9 , there exists a nondecreasing sequence $\left\{m_{k}\right\} \subset \mathbf{N}$ such that $m_{k} \rightarrow \infty$, and

$$
d^{2}\left(x_{m_{k}}, p\right) \leq d^{2}\left(x_{m_{k}+1}, p\right) \text { and } d^{2}\left(x_{k}, p\right) \leq d^{2}\left(x_{m_{k}+1}, p\right) \forall k \in \mathbf{N} .
$$

Thus, with this and (3.16), we obtain

$$
\begin{aligned}
0 & \leq \lim _{k \rightarrow \infty}\left(d^{2}\left(x_{m_{k}+1}, p\right)-d^{2}\left(x_{m_{k}}, p\right)\right) \\
& \leq \limsup _{n \rightarrow \infty}\left(d^{2}\left(x_{n+1}, p\right)-d^{2}\left(x_{n}, p\right)\right) \\
& \leq \limsup _{n \rightarrow \infty}\left(d^{2}\left(u_{n}, p\right)+\left(k_{n}^{2}-1\right) d^{2}\left(u_{n}, p\right)-d^{2}\left(x_{n}, p\right)\right)
\end{aligned}
$$




$$
\begin{aligned}
& \leq \limsup _{n \rightarrow \infty}\left(\left(1-t_{n}\right) d^{2}\left(x_{n}, p\right)+t_{n} d^{2}(u, p)+\left(k_{n}^{2}-1\right) d^{2}\left(u_{n}, p\right)-d^{2}\left(x_{n}, p\right)\right) \\
& \leq \limsup _{n \rightarrow \infty}\left[t_{n}\left(d^{2}(u, p)-d^{2}\left(x_{n}, p\right)\right)+\left(k_{n}^{2}-1\right) M^{\prime}\right]=0,
\end{aligned}
$$

which implies

$$
\lim _{k \rightarrow \infty}\left(d^{2}\left(x_{m_{k}+1}, p\right)-d^{2}\left(x_{m_{k}}, p\right)\right)=0 .
$$

Following the same line of argument as in Case 1, we can verify that

$$
k \rightarrow \infty \lim \left(t_{m_{k}} d^{2}(u, z)+2\left(1-t_{m_{k}}\right)\left\langle\overrightarrow{u z}, \overrightarrow{x_{n} z}\right\rangle\right) \leq 0 .
$$

Also from (3.36), we have

$$
\begin{gathered}
d^{2}\left(x_{m_{k}+1}, z\right) \leq\left(1-t_{m_{k}}\right) d^{2}\left(x_{m_{k}}, z\right)+t_{m_{k}}\left(t_{m_{k}} d^{2}(u, z)+2\left(1-t_{m_{k}}\right)\left\langle\overrightarrow{u z}, \overrightarrow{x_{m_{k}}} \vec{z}\right\rangle\right) \\
+\left(k_{m_{k}}^{2}-1\right) M^{\prime} .
\end{gathered}
$$

Since $d^{2}\left(x_{m_{k}}, z\right) \leq d^{2}\left(x_{m_{k}+1}, z\right)$, we have

$$
d^{2}\left(x_{m_{k}}, z\right) \leq\left(t_{m_{k}} d^{2}(u, z)+2\left(1-t_{m_{k}}\right)\left\langle\overrightarrow{u z}, \overrightarrow{x_{m_{k}}} \vec{z}\right\rangle\right)+\frac{\left(k_{m_{k}}^{2}-1\right) M^{\prime}}{t_{m_{k}}},
$$

which implies from (3.38) and condition C4 that

$$
\lim _{k \rightarrow \infty} d^{2}\left(x_{m_{k}}, z\right)=0 .
$$

Since $d^{2}\left(x_{k}, z\right) \leq d^{2}\left(x_{m_{k}+1}, z\right)$, we obtain from (3.39) and (3.37) that $k \rightarrow \infty \lim d^{2}\left(x_{k}, z\right)=0$. Thus, from Case 1 and Case 2, we conclude that $\left\{x_{n}\right\}$ converges to $z \in \Gamma$.

If $T$ is a uniformly $L$-Lipschitzian and asymptotically nonexpansive mapping defined on $X$, then we obtain the following result.

Let $X$ be a Hadamard space and $X^{*}$ be its dual space. Let $A_{i}: X \rightarrow$ $2^{X^{*}}, i=1,2, \ldots N$ be multivalued monotone mappings that satisfy the range condition and $f_{j}: X \rightarrow(-\infty, \infty], j=1,2, \ldots, m$ be proper convex and lower semi continuous functions. Let $T: X \rightarrow X$ be a uniformly $L$-Lipschitzian and asymptotically nonexpansive mapping. Suppose that $\Gamma:=F(T) \cap\left(\cap_{i=1}^{N} A_{i}^{-1}(0)\right) \cap\left(\cap_{j=1}^{m} \arg \min _{y \in X} f_{j}(y)\right) \neq \emptyset$. Let $u, x_{1} \in X$ be arbitrary and the sequence $\left\{x_{n}\right\}$ be generated by 


$$
\left\{\begin{array}{l}
v_{n}=\left(1-t_{n}\right) x_{n} \oplus t_{n} u \\
u_{n}=\Phi_{\lambda}^{N} \circ \Psi_{\mu}^{m}\left(v_{n}\right), \\
y_{n}=\left(1-\beta_{n}\right) u_{n} \oplus \beta_{n} T^{n} u_{n} \\
x_{n+1}=\left(1-\alpha_{n}\right) u_{n} \oplus \alpha_{n} T^{n} y_{n}, n \geq 1
\end{array}\right.
$$

where $\Phi_{\lambda}^{N}=J_{\lambda}^{N} \circ J_{\lambda}^{N-1} \circ \ldots \circ J_{\lambda}^{2} \circ J_{\lambda}^{1}, \quad \Phi_{\lambda}^{0}=I, \Psi_{\mu}^{m}=J_{\mu}^{N} \circ J_{\mu}^{m-1} \circ \ldots \circ$ $J_{\mu}^{2} \circ J_{\mu}^{1}, \quad \Phi_{\mu}^{0}=I, \lambda, \mu \in(0, \infty)$ and $\left\{t_{n}\right\}$ is a sequence in $(0,1)$ satisfying the following conditions:

$\mathrm{C} 1: n \rightarrow \infty \lim t_{n}=0$,

$\mathrm{C} 2: \sum_{n=1}^{\infty} t_{n}=\infty$,

C3: $0<\epsilon \leq \alpha_{n} \leq b<1$ and $0<\epsilon \leq \beta_{n} \leq b<1$,

C4: $n \rightarrow \infty \lim \frac{k_{n}^{2}-1}{t_{n}}=0$.

Then $\left\{x_{n}\right\}$ converges strongly to an element of $\Gamma$.

By setting $N=m=1$ in Theorem 3.3, we obtain the following result.

Let $X$ be a Hadamard space and $X^{*}$ be its dual space. Let $A$ : $X \rightarrow 2^{X^{*}}$ be multivalued monotone mapping that satisfies the range condition and $f: X \rightarrow(-\infty, \infty]$ be proper convex and lower semi continuous function. Let $T: X \rightarrow X$ be a uniformly $L$-Lipschitzian and asymptotically demicontractive mapping with constant $k \in(0,1)$ and sequence $\left\{k_{n}\right\}_{n=1}^{\infty} \subseteq[1, \infty)$ such that $\sum_{n=1}^{\infty}\left(k_{n}^{2}-1\right)<\infty$. Suppose that $\Gamma:=F(T) \cap A^{-1}(0) \cap \arg \min _{y \in X} f(y) \neq \emptyset$. Let $u, x_{1} \in X$ be arbitrary and the sequence $\left\{x_{n}\right\}$ be generated by

$$
\left\{\begin{array}{l}
\mathrm{v}_{n}=\left(1-t_{n}\right) x_{n} \oplus t_{n} u \\
\mathrm{u}_{n}=\left(1-J_{\lambda}\right) u_{n} \oplus \beta_{n} T^{n} u_{n} \\
\mathrm{y}_{n}=\left(1-v_{n}\right) \\
\mathrm{x}_{n+1}=\left(1-\alpha_{n}\right) u_{n} \oplus \alpha_{n} T^{n} y_{n}, n \geq 1,
\end{array}\right.
$$

where $\lambda, \mu \in(0, \infty)$ and $\left\{t_{n}\right\},\left\{\beta_{n}\right\},\left\{\alpha_{n}\right\}$ are sequences in $(0,1)$ satisfying the following conditions:

$\mathrm{C} 1: n \rightarrow \infty \lim t_{n}=0$,

$\mathrm{C} 2: \sum_{n=1}^{\infty} t_{n}=\infty$

C3: $0<\epsilon \leq \alpha_{n} \leq k \beta_{n}<\beta_{n} \leq b<\frac{2}{\left(\frac{k+\delta}{k}\right)+\sqrt{\left(\frac{k+\delta}{k}\right)^{2}+4 L^{2}}}$, where $\delta:=$ $n \geq 1 \sup k_{n}$, 
$\mathrm{C} 4: n \rightarrow \infty \lim \frac{k_{n}^{2}-1}{t_{n}}=0$.

Then $\left\{x_{n}\right\}$ converges strongly to an element of $\Gamma$.

By setting $N=m=1$ and $T=I$ ( $I$ is the identity mapping on $X$ ) in Theorem 3.3, we obtain the following result.

Let $X$ be a Hadamard space and $X^{*}$ be its dual space. Let $A: X \rightarrow 2^{X^{*}}$ be multivalued monotone mapping that satisfies the range condition and $f: X \rightarrow(-\infty, \infty]$ be proper convex and lower semi continuous function. Suppose that $\Gamma:=A^{-1}(0) \cap \arg \min _{y \in X} f(y) \neq \emptyset$. Let $u, x_{1} \in X$ be arbitrary and the sequence $\left\{x_{n}\right\}$ be generated by

$$
\left\{\begin{array}{l}
v_{n}=\left(1-t_{n}\right) x_{n} \oplus t_{n} u, \\
u_{n}=\left(J_{\lambda} \circ J_{\mu}\left(v_{n}\right)\right) \\
y_{n}=\left(1-\beta_{n}\right) u_{n} \oplus \beta_{n} u_{n}, \\
x_{n+1}=\left(1-\alpha_{n}\right) u_{n} \oplus \alpha_{n} y_{n}, n \geq 1,
\end{array}\right.
$$

where $\lambda, \mu \in(0, \infty)$ and $\left\{t_{n}\right\},\left\{\beta_{n}\right\},\left\{\alpha_{n}\right\}$ are sequences in $(0,1)$ satisfying the following conditions:

$\mathrm{C} 1: n \rightarrow \infty \lim t_{n}=0$,

$\mathrm{C} 2: \sum_{n=1}^{\infty} t_{n}=\infty$.

Then $\left\{x_{n}\right\}$ converges strongly to an element of $\Gamma$.

\section{Numerical Example}

In this section, we give a numerical example of Theorem 3.3 to illustrate the applicability of our main result. Let $X=\mathbf{R}^{2}$ be endowed with the euclidean norm $\|.\|_{2}$. Let $B: \mathbf{R}^{2} \rightarrow \mathbf{R}^{2}$ be defined by $B(x)=\left(2 x_{1}+x_{2}, x_{1}+3 x_{2}\right)$ and $b^{T}=(0,0)$. Let $m=1$ in Theorem 3.3 , we define $f: \mathbf{R}^{2} \rightarrow(-\infty, \infty]$ by

$$
f(x)=\frac{1}{2}\|B(x)-b\|^{2} .
$$

Then, $f$ is a proper convex and lower semi continuous function, since $B$ is a continuous linear mapping (see [43], [18]). Thus for $\mu=1$, we obtain from [43] that

$$
\begin{aligned}
J_{1}(x)=\operatorname{Prox}_{f}(x) & =\arg \min _{y \in \mathbf{R}^{2}}\left[f(y)+\frac{1}{2}\|y-x\|^{2}\right] \\
& =\left(I+B^{T} B\right)^{-1}\left(x+B^{T} b\right) .
\end{aligned}
$$


So that

$$
\begin{aligned}
J_{1}(x)=\left(\left[\begin{array}{ll}
1 & 0 \\
0 & 1
\end{array}\right]\right. & \left.+\left[\begin{array}{cc}
2 & 1 \\
1 & 3
\end{array}\right]\left[\begin{array}{ll}
2 & 1 \\
1 & 3
\end{array}\right]\right)^{-1}\left(\left[\begin{array}{l}
x_{1} \\
x_{2}
\end{array}\right]+\left[\begin{array}{ll}
2 & 1 \\
1 & 3
\end{array}\right]\left[\begin{array}{l}
0 \\
0
\end{array}\right]\right) \\
& =\left(\frac{11 x_{1}-5 x_{2}}{41}, \frac{-5 x_{1}+6 x_{2}}{41}\right) .
\end{aligned}
$$

Let $N=1$ in Theorem 3.3, we define $A: \mathbf{R}^{2} \rightarrow \mathbf{R}^{2}$ by $A(x)=\left(x_{1}, x_{1}-x_{2}\right)$. Then, $A$ is a monotone mapping.

We note that $[t \overrightarrow{a b}] \equiv t(b-a)$, for all $t \in \mathbf{R}$ and $a, b \in \mathbf{R}^{2}$ (see [3]). Thus, we have for each $x \in \mathbf{R}^{2}$ that

$$
\begin{aligned}
J_{\lambda}(x)=z & \Longleftrightarrow \frac{1}{\lambda}(x-z) \in A z \\
& \Longleftrightarrow x=(I+\lambda A) z \\
& \Longleftrightarrow z=(I+\lambda A)^{-1} x .
\end{aligned}
$$

So that for $\lambda=2$, we compute the resolvent of $A$ as follows:

$$
\begin{gathered}
J_{2}(x)=\left(\left[\begin{array}{ll}
1 & 0 \\
0 & 1
\end{array}\right]+2\left[\begin{array}{cc}
1 & 0 \\
1 & -1
\end{array}\right]\right)^{-1}\left[\begin{array}{l}
x_{1} \\
x_{2}
\end{array}\right] \\
=\left(\frac{x_{1}}{5}, \frac{2 x_{1}-3 x_{2}}{5}\right) .
\end{gathered}
$$

Thus, for $N=m=1, \lambda=2$ and $\mu=1$, we obtain

$$
J_{2}\left(J_{1} x\right)=\left(\frac{11 x_{1}-5 x_{2}}{205}, \frac{37 x_{1}-28 x_{2}}{205}\right) \text {. }
$$

Let $T: \mathbf{R}^{2} \rightarrow \mathbf{R}^{2}$ be defined by $T(x)=\frac{-3 x}{2}$. Then $T$ is a demicontractive mapping with $k=\frac{1}{5}$. This implies that $\frac{2}{T}$ is an asymptotically demicontractive mapping with $k=\frac{1}{5}$ and $k_{n}=1$ for all $n \geq 1$. Thus, we obtain that $\delta=1$. It is easy to see that $T$ is also uniformly $L$-Lipschitzian with $L=\frac{3}{2}$. Now, if we take $b=\frac{1}{10}$ and $\epsilon=\frac{1}{1000}$, then condition C3 becomes:

$$
0<\frac{1}{1000} \leq \alpha_{n} \leq \frac{\beta_{n}}{5}<\beta_{n} \leq \frac{1}{10}<\frac{2}{6+\sqrt{45}} .
$$

So that, if we choose $\beta_{n}=\frac{1}{10+\frac{1}{n}}, \alpha_{n}=\frac{1}{50+\frac{5}{n}}$ and $t_{n}=\frac{1}{2 n+1}$, then $t_{n}, \beta_{n}$ and $\alpha_{n}$ satisfy the conditions in Theorem 3.3.

Hence, for $u, x_{1} \in \mathbf{R}^{2}$, our Algorithm (3.12) becomes: 
$(4.1)$

$$
\left\{\begin{array}{l}
v_{n}=\frac{2 n}{2 n+1} x_{n}+\frac{u}{2 n+1}, \\
u_{n}=J_{2}\left(J_{1} v_{n}\right) \\
y_{n}=\left(1-\frac{1}{10+\frac{1}{n}}\right) u_{n}-\frac{3}{20+\frac{2}{n}} u_{n}, \\
x_{n+1}=\left(1-\frac{1}{50+\frac{5}{n}}\right) u_{n}-\frac{3}{100+\frac{10}{n}} y_{n}, n \geq 1 .
\end{array}\right.
$$

Case I Take $x_{1}=(0.1,0.5)^{T}$ and $u=(0.1,0.5)^{T}$.

Case II Take $x_{1}=(0.1,0.5)^{T}$ and $u=(1,1.5)^{T}$.

Case III Take $x_{1}=(-1,-0.5)^{T}$ and $u=(-1,1.5)^{T}$.
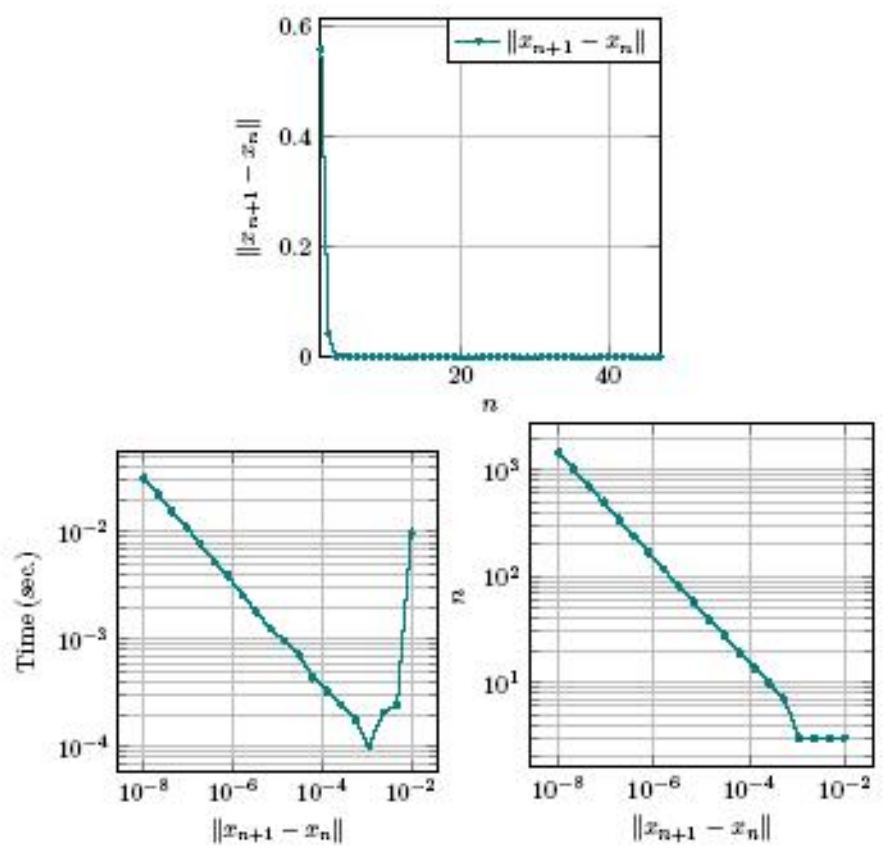

Figure 1. Case I: errors vs number of iterations (top); execution time vs accuracy (bottom left); number of iterations vs accuracy (bottom right). 


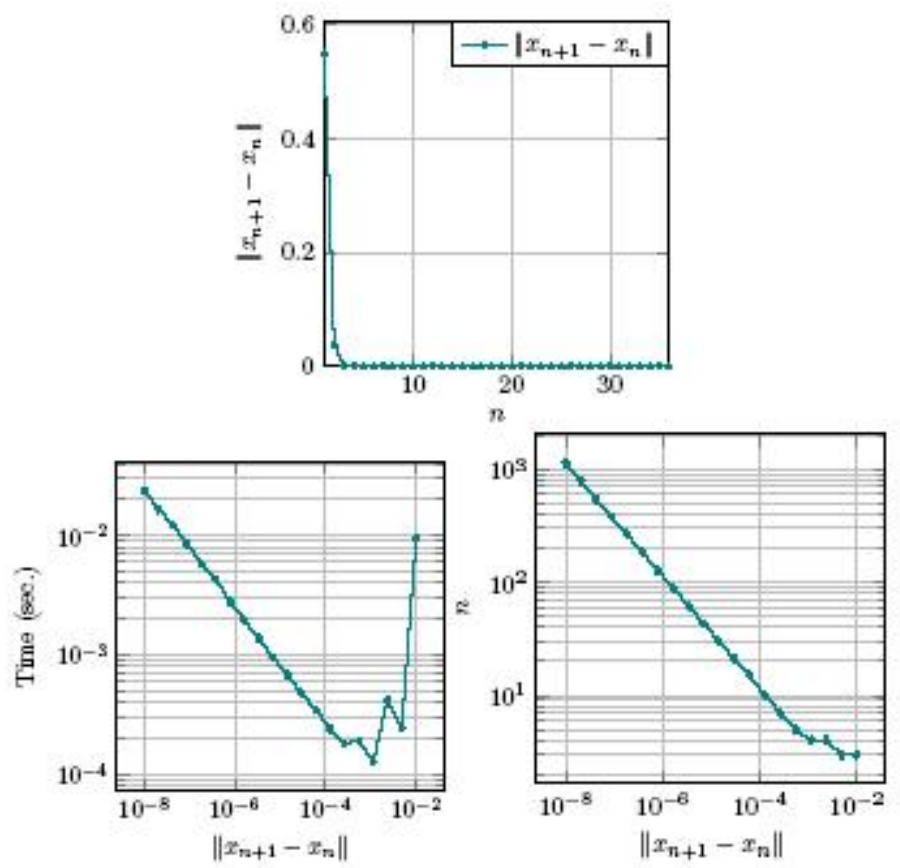

Figure 2. Case II: errors vs number of iterations (top); execution time vs accuracy (bottom left); number of iterations vs aocuracy (bottom right).
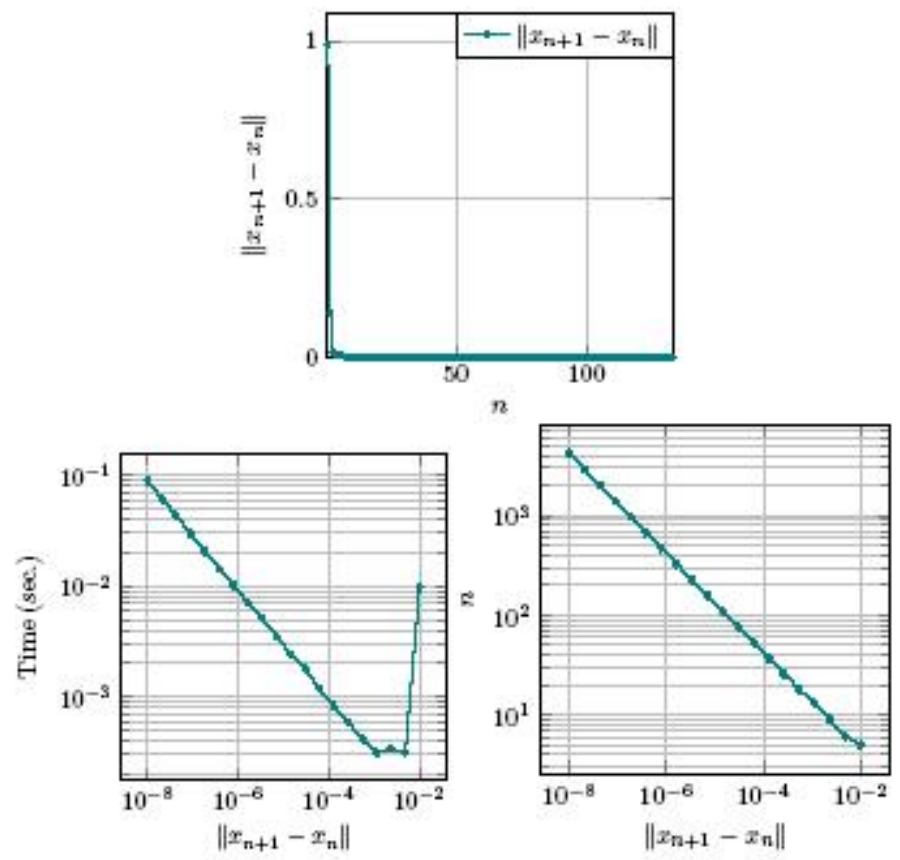

FIGURE 3. Case III: errors vs number of iterations (top); execution time vs accuracy (bottom left); number of iterations vs accuracy (bottom right).

The Mathlab version used is R2014a and the figures are presented in Figure 1, Figure 2 and Figure 3. 


\section{Acknowledgement}

The first author acknowledges with thanks the bur- sary and financial support from Department of Science and Innovation and National Research Foundation, Republic of South Africa Center of Ex-cellence in Mathematical and Statistical Sciences (DSI-NRF CoE-MaSS) Doctoral Bursary. The third author is supported in part by the National Research Foundation (NRF) of South Africa Incentive Funding for Rated Researchers (Grant Number 119903). Opinions expressed and conclusions arrived are those of the authors and are not necessarily to be attributed to the CoE-MaSS and NRF.

\section{References}

[1] H. A. Abass, K. O. Aremu, L. O. Jolaoso and O. T. Mewomo, "An inertial forward-backward splitting method for approximating solutions of certain optimization problems", Journal of nonlinear functional analysis, vol. 2020, Art. ID 6, 2020, doi: 10.23952/ jnfa.2020.6

[2] T. O. Alakoya, L. O. Jolaoso, and O. T. Mewomo, "Modified inertia subgradient extragradient method with self adaptive stepsize for solving monotone variational inequality and fixed point problems", Optimization, vol. 70, no. 3, pp. 545-572, 2021, doi: 10.1080/ 02331934.2020.1723586

[3] B. A. Kakavandi and M. Amini, "Duality and subdifferential for convex functions on complete CAT(0) metric spaces", Nonlinear analysis: theory, methods \& applications, vol. 73, no. 10, pp. 3450-3455, 2000, doi: 10.1016/j.na.2010.07.033

[4] K. O. Aremu, H. A. Abass, C. Izuchukwu, and O. T. Mewomo, "A viscosity-type algorithm for an infinitely countable family of ( $f$, g)-generalized k-strictly pseudononspreading mappings in $\operatorname{CAT}(0)$ spaces", Analysis, vol. 40, no. 1, pp. 19-37, 2020, doi: 10.1515/ anly-2018-0078

[5] K. O. Aremu, C. Izuchukwu, G. N. Ogwo, and O. T. Mewomo, "Multi-step Iterative algorithm for minimization and fixed point problems in p-uniformly convex metric spaces", Journal of industrial \& management optimization, First on line, 2020, doi:10.3934/jimo.2020063

[6] K. O. Aremu, C. Izuchukwu, G. C. Ugwunnadi, and O. T. Mewomo, "On the proximal point algorithm and demimetric mappings in $\operatorname{CAT}(0)$ spaces", Demonstratio mathematica, vol. 51, no. 1, pp. 277-294, 2018, doi: 10.1515/ dema-2018-0022 
[7] D. Ariza-Ruiz, L. Leuştean, G. López-Acedo, "Firmly nonexpansive mappings in classes of geodesic spaces", Transaction of the American Mathematical Society, vol. 366, pp. 4299-4322, 2014, doi: 10.1090/ S0002-9947-2014-05968-0

[8] M. Bačák, "Computing medians and means in Hadamard spaces", SIAM journal on optimization, vol. 24, no. 3, pp. 1542-1566, 2014, doi: $10.1137 / 140953393$

[9] M. Bačák, "The proximal point algorithm in metric spaces", Israel journal of mathematics, vol. 194, pp. 689-701, 2013, doi: 10.1007/ s11856-012-0091-3

[10] M. Bačák and S. Riech, "The asymptotic behavior of class of nonlinear semigroup in Hadamard spaces", Journal of fixed point theory and applications, vol. 16, pp. 189-202, 2014, doi: 10.1007/ s11784-014-0202-3

[11] I. D. Berg and I.G. Nikolaev, "Quasilinearization and curvature of Alexandrov spaces", Geometriae dedicate, vol. 133, pp. 195-218, 2008, doi: $10.1007 /$ s10711-008-9243-3

[12] M. R. Bridson and A. Haefliger, Metric spaces of non-positive curvature. Berlin: Springer, 1999, doi: 10.1007/978-3-662-12494-9

[13] R. E. Bruck and S. Reich, "Nonexpansive projections and resolvents of accretive operators in Banach spaces", Houston journal of mathematics, vol. 3, no. 4, pp. 459-470, 1977.

[14] F. Bruhat and J. Tits, "Groupes réductifs sur un corp local", Publications Mathématiques de l'Institut des Hautes Études Scientifiques, vol. 41, pp. 5-251, 1972, doi: 10.1007/ BF02715544

[15] C. Byrne, Y. Censor, A. Gibali, and S. Reich, "The split common null point problem", Journal of nonlinear and convex analysis, vol. 13, no. 4, pp. 759-775, 2012.

[16] P. Chaoha, and A. Phon-on, "A note on fixed point sets in $\operatorname{CAT}(0)$ spaces", Journal of mathematical analysis and applications, vol. 320, no. 2, pp. 983-987, 2006, doi: 10.1016/j.jmaa.2005.08.006

[17] C. E. Chidume, and C. O. Chidume, "Iterative approximation of fixed points of nonexpansive mappings", Journal of mathematical analysis and applications, vol. 318, no. 1, pp. 288-295, 2006, doi: 10.1016/j.jmaa.2005.05.023

[18] P. L. Combettes, and J. C. Pesquet, "Proximal splitting methods in signal processing”, May 2010. arXiv:0912.3522v4

[19] H. Dehghan, and J. Rooin, "Metric projection and convergence theorems for nonexpansive mapping in Hadamard spaces", Oct. 2014. arXiv: $1410.1137 \mathrm{v} 1$ 
[20] S. Dhompongsa, W. A. Kirk, B. Panyanak, "Nonexpansive set-valued mappings in metric and Banach spaces", Journal of nonlinear and convex analysis, vol. 8, no. 1, pp. 35-45, 2007.

[21] S. Dhompongsa, W.A. Kirk, and B. Sims, "Fixed points of unifromly Lipschitzian mappings", Nonlinear analysis: theory, methods \& applications, vol. 65, no. 4, pp. 762-772, 2006, doi: 10.1016/j.na.2005.09.044

[22] K. Göbel and S. Reich, Uniform convexity, hyperbolic geometry, and nonexpansive mappings. New York, NY: Dekker, 1984.

[23] O. Güler, "On the convergence of the proximal point algorithm for convex minimization", SIAM journal on control and optimization, vol. 29, no. 2, pp. 403-419, 1991, doi: 10.1137/0329022

[24] C. Izuchukwu, G. C. Ugwunnadi, O. T. Mewomo, A. R. Khan, and M. Abbas, "Proximal-type algorithms for split minimization problem in p-uniformly convex metric space", Numerical algorithms, vol. 82, no. 3, pp. 909-935, 2019, doi: 10.1007/ s11075-018-0633-9

[25] C. Izuchukwu, K. O. Aremu, A. A. Mebawondu, and O. T. Mewomo, "A viscosity iterative technique for equilibrium and fixed point problems in Hadamard space", Applied general topology, vol. 20, no. 1, pp. 193-210, 2019, doi: 10.4995/ agt.2019.10635

[26] L.O. Jolaoso, T. O. Alakoya, A. Taiwo, and O. T. Mewomo, "A parallel combination extragradient method with Armijo line searching for finding common solution of finite families of equilibrium and fixed point problems", Rendiconti del Circolo Matematico di Palermo Series 2, vol. 69, pp. 711-735, 2020, doi: 10.1007/ s12215-019-00431-2

[27] L. O. Jolaoso, F. U. Ogbuisi, and O. T. Mewomo, "An iterative method for solving minimization, variational inequality and fixed point problems in reflexive Banach spaces", Advances in pure and applied mathematics, vol. 9, no. 3, pp. 167-184, 2018, doi: 10.1515/ apam-2017-0037

[28] L.O. Jolaoso, O.K. Oyewole, C.C. Okeke, and O. T. Mewomo, "A unified algorithm for solving split generalized mixed equilibrium problem and fixed point of nonspreading mapping in Hilbert space", Demonstratio mathematica, vol. 51, no. 1, pp 211-232, 2018, doi: 10.1515/ dema-2018-0015

[29] L.O. Jolaoso, A. Taiwo, T.O. Alakoya, and O. T. Mewomo, "A self adaptive inertial subgradient extragradient algorithm for variational inequality and common fixed point of multivalued mappings in Hilbert spaces", Demonstratio mathematica, vol. 52, no. 1, pp. 183-203, 2019, doi: 10.1515/ dema-2019-0013 
[30] L. O. Jolaoso, A. Taiwo, T.O. Alakoya, and O. T. Mewomo, "A unified algorithm for solving variational inequality and fixed point problems with application to the split equality problem", Computational and applied mathematics, vol. 39, Art ID. 38, 2019, doi: 10.1007/ s40314-019-1014-2

[31] L. O. Jolaoso, A. Taiwo, T. O. Alakoya, and O. T. Mewomo, "Strong convergence theorem for solving pseudo-monotone variational inequality problem using projection method in a reflexive Banach space", Journal of optimization theory and applications, vol. 185, pp. 744-766, 2020, doi: 10.1007/ s10957-020-01672-3

[32] J. Jost, "Convex functionals and generalized harmonic maps into spaces of nonpositive curvature", Commentarii mathematici helvetici, vol. 70, pp. 659-673, 1995, doi: 10.1007/ BF02566027

[33] B. A Kakavandi and M. Amini, "Duality and subdifferential for convex functions on complete CAT(0) metric spaces", Nonlinear analysis: theory, methods \& applications, vol. 73, no. 10, 3450-3455, 2010, doi: 10.1016/j.na.2010.07.033

[34] S. Kamimura and W. Takahashi, "Approximating solutions of maximal monotone operators in Hilbert spaces", Journal of approximation theory, vol. 106, no. 2, pp. 226-240, 2010, doi: 10.1006/ jath.2000.3493

[35] K. R. Kazmi and S. H. Rizvi, "An iterative method for split variational inclusion problem and fixed point problem for a nonexpansive mapping", Optimization letters, vol. 8, no. 3, 2014, doi: 10.1007/ s11590-013-0629-2

[36] H. Khatibzadeh and S. Ranjbar, "Monotone operators and the proximal point algorithm in complete CAT(0) metric spaces", Journal of the Australian Mathematical Society, vol. 103, no. 1, pp. 70-90, 2017, doi:101017S1446788716000446

[37] K. S. Kim, "Some convergence theorems for contractive type mappings in CAT(0) spaces", Abstract and applied analysis, vol. 2013, Art. ID. 381715, 2013, doi: 10.1155/2013/381715

[38] W. A. Kirk, "Geodesic geometry and fixed point theory," in Seminar of mathematical analysis. Proceedings, Universities of Malaga and Seville (Spain), September 2002-February 2003, 2003, pp. 195-225.

[39] W. A. Kirk and B. Panyanak, "A concept of convergence in geodesic spaces", Nonlinear analysis: theory, methods \& applications, vol. 68, no. 12, pp. 3689-3696, 2008, doi: 10.1016/ j.na.2007.04.011

[40] W. Laowang and B. Panyanak, "Strong and $\Delta$-convergence theorems for multivalued mappings in CAT(0) spaces", Journal of inequalities and applications, vol. 2009, Art. ID 730132, 2009, doi: $10.1155 / 2009 / 730132$ 
[41] L. Leustean, "Nonexpansive iterations uniformly cover W-hyperbolic spaces," in Nonlinear analysis and optimization I: nonlinear analysis, A. Leizarowitz, B. S. Mordukhovich, I. Shafrir, and A. J. Zaslavski, Eds. Providence, RI: American Mathematical Society, 2010, pp. 193-209, doi: 10.1090/ conm/ 513/ 10084

[42] T. C. Lim, "Remarks on some fixed point theorems", Proceedings of the American Mathematical Society, vol. 60, pp. 179-182, 1976, doi: 10.1090/ S0002-9939-1976-0423139-X

[43] B. Martinet, “Régularisation d'Inéquations variationnelles par approximations successives", Revue française d'informatique et de recherche opérationnelle. Série rouge, vol. 3, no. R3, pp. 154-158, 1970, doi: 10.1051/ m2an/ 197004R301541

[44] P. E. Maingé, "Strong convergence of projected subgradient methods for nonsmooth and nonstrictly convex minimization", Set-valued analysis, vol. 16, pp. 899-912, 2008, doi: 10.1007/s11228-008-0102-z

[45] J. Merryfield and J. D. Stein, "A generalization of the Banach contraction principle", Journal of mathematical analysis and applications, vol. 273, no. 1, pp. 112-120, 2002, doi: 10.1016/ S0022-247X(02)00215-9

[46] O. Nevanlinna and S. Reich, "Strong convergence of contraction semigroups and of iterative methods for accretive operators in Banach spaces", Israel journal of mathematics, vol. 32, pp. 44-58, 1979, doi: 10.1007/ BF02761184

[47] G. N. Ogwo, C. Izuchukwu, K. O. Aremu, and O. T. Mewomo, "A viscosity iterative algorithm for a family of monotone inclusion problems in an Hadamard space", Bulletin of the Belgian Mathematical Society - Simon Stevin, vol. 27, no. 1, pp. 127-152, 2020, doi: $10.36045 / \mathrm{bbms} / 1590199308$

[48] F. U. Ogbuisi and O. T. Mewomo, "Iterative solution of split variational inclusion problem in real Banach space", Afrika matematika, vol. 28, no. 2, pp. 295-309, 2017, doi: 10.1007/ s13370-016-0450-z

[49] O. K. Oyewole, H. A. Abass, and O.T. Mewomo, "Strong convergence algorithm for a fixed point constraint split null point problem", Rendiconti del Circolo Matematico di Palermo Series 2, vol. 2020, 2020, doi: 10.1007/ s12215-020-00505-6

[50] S. Ranjbar and H. Khatibzadeh, "Strong and $\Delta$-convergence to a zero of a monotone operator in CAT(0) spaces", Mediterranean journal of mathematics, vol. 14, Art. ID. 56, 2017, doi:10.1007/ s00009-017-0885-y

[51] S. Reich, and I. Shafrir, "Nonexpansive iterations in hyperbolic spaces", Nonlinear analysis: theory, methods \& applications, vol. 15, no. 6, pp. 537-558, 1990, doi: 10.1016/ 0362-546X(90)90058-O 
[52] R. T., Rockafellar, "Monotone operators and the proximal point algorithm", SIAM journal on control and optimization, vol. 14, no. 5, pp. 877-898, 1976, doi: 10.1137/0314056

[53] Y. Shehu and O. T. Mewomo, "Further investigation into split common fixed point problem for demicontractive operators", Acta mathematica sinica, english series, vol. 32, no. 11, pp. 1357-1376, 2016, doi: 10.1007/ s10114-016-5548-6

[54] R. Suparatulatorn, P. Cholamjiak, and S. Suantai, "On solving the minimization problem and the fixed-point problem for nonexpansive mappings in CAT(0) spaces", Optimization methods and software, vol. 32, no. 1, pp. 182-192, 2016, doi: 10.1080/ 10556788.2016.1219908

[55] A. Taiwo, T. O. Alakoya, and O.T. Mewomo, "Halpern-type iterative process for solving split common fixed point and monotone variational inclusion problem between Banach spaces", Numerical algorithms, vol. 2020, doi: 10.1007/ s11075-020-00937-2

[56] A. Taiwo, L. O. Jolaoso, and O. T. Mewomo, "A modified Halpern algorithm for approximating a common solution of split equality convex minimization problem and fixed point problem in uniformly convex Banach spaces", Computational and applied mathematics, vol. 38, no. 2, Art. ID. 77, 2019, doi: 10.1007/ s40314-019-0841-5

[57] A. Taiwo, L. O. Jolaoso, and O. T. Mewomo, "Parallel hybrid algorithm for solving pseudomonotone equilibrium and split common fixed point problems", Bulletin of the Malaysian Mathematical Sciences Society, vol. 43, pp. 1893-1918, 2020, doi: 10.1007/ s40840-019-00781-1

[58] A. Taiwo, L. O. Jolaoso, and O. T. Mewomo, "Viscosity approximation method for solving the multiple-set split equality common fixed-point problems for quasi-pseudocontractive mappings in Hilbert spaces", Journal of industrial \& management optimization, First on line, 2020, doi:10.3934/jimo.2020092

[59] G. C. Ugwunnadi, C. Izuchukwu, and O. T. Mewomo, "Strong convergence theorem for monotone inclusion problem in $\mathrm{CAT}(0)$ spaces", Afrika matematika, vol. 30, no. 1-2, pp. 151-169, 2019, doi: 10.1007/ s13370-018-0633-x

[60] G. C. Ugwunnadi, C. Izuchukwu, and O. T. Mewomo, "Proximal point algorithm involving fixed point of nonexpansive mapping in p-uniformly convex metric space", Advances in pure and applied mathematics, vol. 10, no. 4, pp. 437-446, 2019, doi: 10.1515/ apam-2018-0026

[61] H. K. Xu, "Iterative algorithms for nonlinear operators", Journal of the London Mathematical Society, vol. 66, no. 1, pp. 240-256, 2012, doi: 10.1112/ S0024610702003332 